\title{
A robust approach to model densification and crack formation in powder compaction processes
}

\author{
J. A. Hernández ${ }^{1,2, *, \dagger}$, J. Oliver ${ }^{3}$, J. C. Cante ${ }^{2}$ and R. Weyler ${ }^{2}$ \\ ${ }^{1}$ International Center for Numerical Methods in Engineering (CIMNE), Technical University of Catalonia, \\ Edificio C1, Campus Norte, Jordi Girona 1-3, Barcelona 08034, Spain \\ ${ }^{2}$ E.T.S. d'Enginyeries Industrial i Aeronàutica de Terrassa, Technical University of Catalonia, C/Colom, 11, \\ Terrassa 08222, Spain \\ ${ }^{3}$ E.T.S. d'Enginyers de Camins, Canals i Ports, Technical University of Catalonia, Edificio C1, Campus Norte, \\ Jordi Girona 1-3, Barcelona 08034, Spain
}

\begin{abstract}
SUMMARY
This paper deals with the question of how to efficiently integrate a constitutive model that describes the densification of powders and the potential formation of cracks in Powder Metallurgy $(\mathrm{P} / \mathrm{M})$ cold compaction processes. The analyzed model is a large strain, elastoplastic model of the Drucker-Prager/Cap type, refined to cover also the prediction of crack formation, and featuring non-conventional elements such as a densitydependent Von Mises yield surface; a parabolic plastic potential function for the Drucker-Prager envelope; and a softening law whose softening modulus is dependent on the level of densification. The employed integration procedure is a non-conventional hybrid or IMPLicit-EXplicit (IMPL-EX) scheme, whose essence is to solve explicitly for some variables and implicitly for others, with the peculiarity of the 'explicit' variables being but extrapolated values of the same quantities computed, at previous time steps, by means of a fully implicit scheme. The return-mapping equations stemming from this implicit scheme are solved using an unconditionally convergent, fractional step method-based iterative procedure. The performance of the IMPL-EX integration algorithm is critically assessed in two different situations: the densification of a cylindrical specimen, and the fracture process in a diametral compression test. Results obtained show conclusively that the proposed hybrid integration strategy offers an efficient solution to the trade-off between robustness and computational time requirements. Copyright (C) 2011 John Wiley \& Sons, Ltd.
\end{abstract}

Received 2 September 2010; Revised 15 December 2010; Accepted 16 December 2010

KEY WORDS: IMPL-EX integration method; fractional step method; softening; powder metallurgy; cracking

\section{INTRODUCTION}

Powder metallurgy $(\mathrm{P} / \mathrm{M})$ is a forming technology aimed at achieving net or near-net parts by compacting metal powders into a discrete shape, and subsequently heating the preform to a temperature below its melting point (sintering). To deem successful a given compaction route, the compacted part, usually referred to as 'compact' or 'green compact', has to meet two major quality requirements: (I) density distribution must be as uniform as possible, to avoid uneven shrinkage in the subsequent sintering; (II) the compact must be free of cracks or other mechanical defects that may prove eventually detrimental to the efficient performance of the finished part. These quality requirements indicate clearly the features of the material response that a numerical model should capture to be a meaningful representation of the industrial process. Not only must it be

\footnotetext{
${ }^{*}$ Correspondence to: J. A. Hernández, International Center for Numerical Methods in Engineering (CIMNE), Technical University of Catalonia, Edificio C1, Campus Norte, Jordi Girona 1-3, Barcelona 08034, Spain.

†E-mail: jhortega@cimne.upc.edu
} 
able to predict final density distributions and estimate with a reasonable accuracy the increase of strength induced by densification; it must also consider the eventual initiation and growth of cracks caused by inappropriate ejection schemes or improper tooling deflections, a task by no means trivial, since densification and cracking, both paradigms of non-linear material behavior, are also intimately connected phenomena: the higher the density attained locally, the higher the strength and the toughness, and hence the resistance to the propagation of cracks.

The first author has proposed elsewhere [1] a large strain, elastoplastic constitutive model able to describe, in a unified manner, these conspicuously distinct phenomena. The model is basically an extension of the classical Drucker-Prager/Cap type model, which is refined to cover also the description of material failure. The purpose of this paper is to discuss in-depth how to efficiently integrate in time this type of constitutive model.

A review of the $\mathrm{P} / \mathrm{M}$ compaction modeling literature reveals that, in selecting the method for integrating the constitutive rate equations, $\mathrm{P} / \mathrm{M}$ modelers have largely favored the explicit forward Euler scheme [2-7] over the implicit backward Euler method [8,9] and other more complex procedures [10]. The reason behind the popularity of explicit solutions is well known: easiness of implementation. In implicit methods, the convergence of the Newton-Raphson iterative solution for the non-linear equilibrium equation is inescapably bound up with the spectral properties of the resulting tangent stiffness matrix, which, in turn, depends crucially on the positive-definite character of such an algorithmic tangent operator. Owing to the aforementioned highly non-linear, tightly coupled nature of the constitutive equations, the derivation of a closed-form expression for the algorithmic tangent operator consistent with the fully implicit scheme is, on its own, an exceedingly complicated task. Furthermore, even in the case of having at one's disposal this algorithmic tangent operator, the lack of positive definiteness caused by strain softening - an indispensable element to
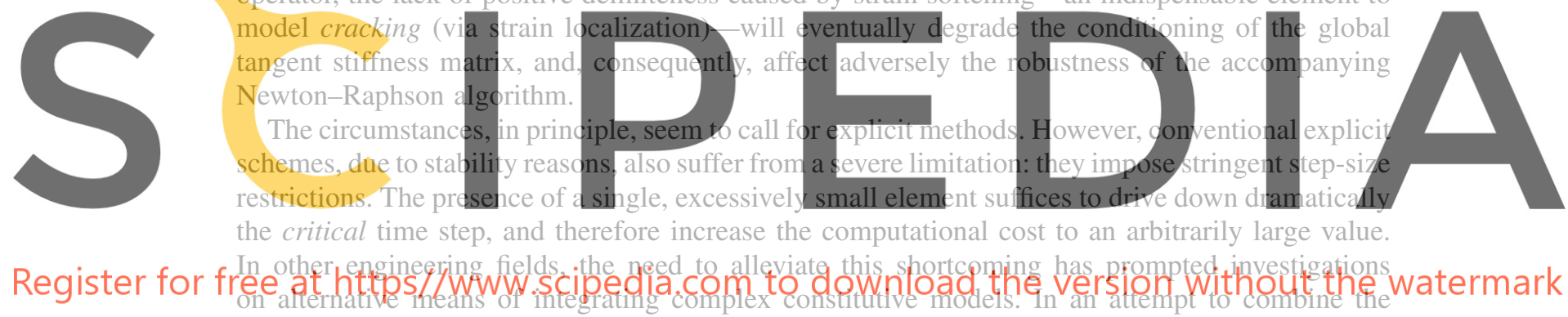

strengths of both conventional implicit and explicit methods, and inspired by the success of the so-called semi-implicit methods for the solution of the equations of motion in computational fluid mechanics [11], several authors have developed hybrid integration schemes [12-16]. The qualifier 'hybrid' means that these methods do not fall neatly in either the categories 'implicit' or 'explicit', but rather share some features of both procedures. They are implicit in the sense that the update of some variables involves the solution of a system of equations; the explicit character comes from the fact that other variables, by contrast, are presumed known or obtained explicitly at the beginning of the time increment.

In this work, we have modified and adapted to the problem under consideration one of these hybrid integration schemes; specifically, the so-called IMPL-EX (IMPLicit-EXplicit) scheme proposed by Oliver et al. $[15,17]$, and originally conceived for overcoming the abovementioned softening-induced robustness problems encountered in the simulation of material failure. The distinguishing feature of this hybrid integration scheme is the manner in which the 'explicit' variables - those whose values are presumed known at the beginning of the time increment-are calculated. Rather than updating these variables by using the pertinent explicit difference equations, as done, for instance, in [12]; or by simply freezing them and a posteriori performing the update, as suggested in [16], the IMPL-EX methodology obtains these values as extrapolations of the same quantities calculated, at previous time steps, by accomplishing a fully implicit integration. According to Oliver et al. (see Reference [17]), this strategy permits to retain the remarkable stability properties exhibited by fully implicit solutions, while at the same time eliminating the robustness deficiencies caused by strain softening. 
In contrast to other semi-implicit methods, thus, the IMPL-EX integration procedure does actually entail the solution of the non-linear system of equations stemming from the implicit backward Euler, the so-called return-mapping equations. This task also conceals many algorithmic challenges, mainly because of, again, the presence of softening. Indeed, the closest point projection method and the cutting-plane algorithm are well-established methods for solving these equations [18]. However, they can only be applied with confidence in the case plasticity models that only feature hardening rules; convergence of the local accompanying iterative scheme (normally a Newton-Raphson algorithm) is not guaranteed when softening comes into play, since the problem ceases to fulfill the required convexity conditions. The related literature abounds with references to strategies that avoid these difficulties (for a fairly comprehensive review, see Armero and PérezFoguet $[19,20]$ and references therein); however, these strategies are specific to particular models, and their generalization is still an open problem.

Therefore, motivated by the inherent limitations of conventional procedures and the ad hoc character of the amendments proposed in the literature, we have developed a return-mapping algorithm especially adanted to the constitutive model under consideration. The essence of the proposed algorithm lies in the decoupling of the evolution equations for the plastic strains and the internal variables, after the fashion of fractional step methods (see e.g. Reference [21]). It will be proved analytically that this method converges to the solution regardless of both the value of the material parameters and the location of the elastic trial stress with respect to the prevailing yield surfaces.

The paper is organized as follows. In Section 2, the constitutive laws and evolution equations developed in Reference [1] are conveniently summarized. The basis of the algorithm for solving the return-mapping equations are presented in Section 3; the mathematical formalisms behind the issues of the existence and uniqueness of solution of such equations are thoroughly discussed in Appendix A. In Sedtion 4, the stress uparte and algorithmic tangent moduli expressions stemming from the IMPL-EX tion of the algorith the numerical asses are provided in the
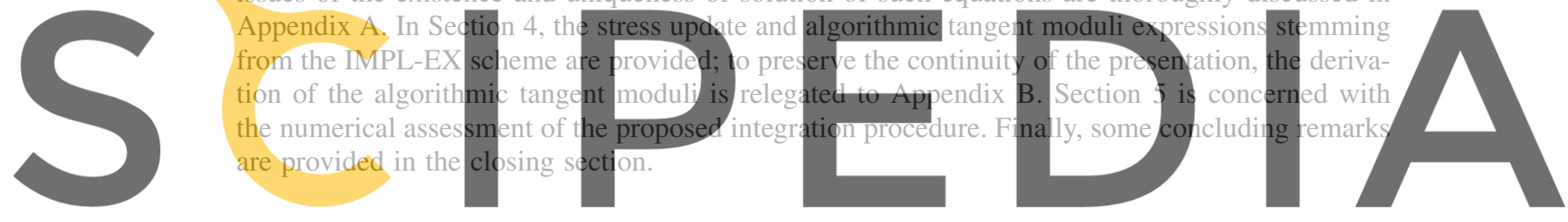

Register for free at https//www.scipedia.Comptonddumffosad the version without the watermark

Consider the powder contained in the die cavity as a deformable body $\mathscr{B}$ consisting of continuously distributed material occupying, at a reference time $t_{0}$, a region $\Omega_{0}$ of $\mathbb{R}^{n}(n=2,3)$. The deformation at time $t$ relative to the reference configuration is given by an one-to-one $\mathscr{C}^{2}$ mapping $\varphi: \Omega_{0} \rightarrow$ $\Omega_{t} \subset \mathbb{R}^{n}$. Reference [1] is entirely devoted to formulate a system of constitutive laws and evolution equations that describes mathematically the effects that a given deformation history

$$
\hat{t} \mapsto \mathbf{F}_{\hat{t}}(\mathbf{X})=\mathbf{F}(\mathbf{X}, \hat{t}) \quad \text { where } \hat{t} \in\left[t_{0}, t\right]
$$

( $\mathbf{F}$ denoting the deformation gradient of $\boldsymbol{\varphi}$ ) has on the physical attributes of the powder at a point $\mathbf{X} \in \Omega_{0}$ (a quadrature point in typical finite element implementations). In the following, we summarize and discuss briefly the most relevant aspects of this constitutive model.

\subsection{Kinematics and internal variables}

The classical assumption of the local multiplicative decomposition of $\mathbf{F}$ into plastic and elastic parts is adopted. The kinematic description considers arbitrarily large plastic deformations and small elastic strains. Under such conditions, the Almansi strain tensor $\mathbf{e}$ and the rate of deformation tensor $\mathbf{d}$ inherit the additive structure of classical small strain formulations

$$
\mathbf{e}=\mathbf{e}_{\mathrm{e}}+\mathbf{e}_{\mathrm{p}}, \quad \mathbf{d}=\mathbf{d}_{\mathrm{e}}+\mathbf{d}_{\mathrm{p}},
$$

where the subscripts 'e' and 'p' denote the elastic and plastic parts, respectively. 
The distinct irreversible processes occurring at the microscopic level are macroscopically described in terms of two strain-like, scalar internal variables: an internal hardening variable $\left(\xi^{h}\right)$, associated with the accumulated compressive (plastic) strain, and an internal softening variable $\left(\xi^{s}\right)$, dependent on the accumulated (plastic) shear strain. The formal definition of both $\xi^{h}$ and $\xi^{s}$ is deferred to Section 2.4.

\subsection{Elastic strain-stress relationship}

Owing to the smallness of the elastic strains, the stress-elastic strains constitutive law is borrowed from that of the classical infinitesimal theory

$$
\tau=c_{e}: \mathbf{e}_{\mathrm{e}},
$$

where $\tau$ denotes the Kirchhoff stress tensor and $\boldsymbol{c}_{\boldsymbol{e}}$ stands for the fourth-order elasticity tensor in the current configuration; under the assumption of isotropy, the expression for $\boldsymbol{c}_{\boldsymbol{e}}$ reduces to

$$
c_{e}=\kappa^{e}\left(\xi^{h}\right) \mathbb{1} \otimes \mathbb{1}+2 \mu^{e}\left(\xi^{h}\right)\left(I_{\mathrm{sym}}-\frac{1}{3} \mathbb{1} \otimes \mathbb{1}\right)
$$

where 1 denotes the second-order identity tensor; $\boldsymbol{I}_{\text {sym }}$ symbolizes the symmetric fourth-order identity tensor, and $\kappa^{e}=E^{e}\left(\xi^{h}\right) /\left(3\left(1-2 v^{e}\right)\right)$ and $\mu^{e}=E^{e}\left(\xi^{h}\right) /\left(2\left(1+v^{e}\right)\right)$ the bulk modulus and shear modulus, respectively. These elastic parameters are not constant, but depend on the hardening internal variable through the Young's modulus $E^{e}$

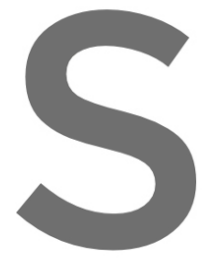

$$
E^{e}\left(\xi^{h}\right)=\frac{\eta_{0}}{\xi^{h}} E_{0} \mathrm{e}^{B_{E}\left(\xi^{h}-\eta_{0}\right)}
$$

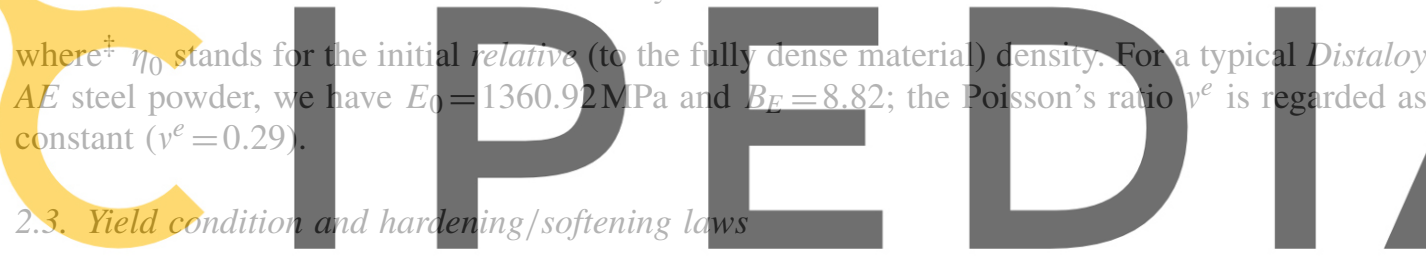

The elastic domain in the Kirchhoff stress space is bounded by three yield surfaces that intersect

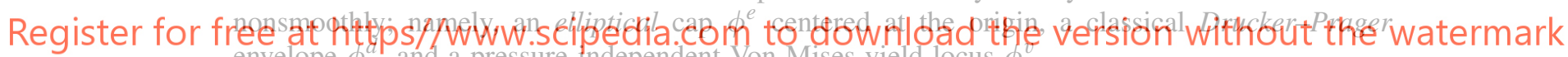
envelope $\phi^{\prime}$, and a pressure-Independent Von Mises yield locus $\phi$

$$
\begin{aligned}
& \phi^{e}=\phi^{1}=q^{2}+s_{2}^{2}\left(\xi^{h}\right) p^{2}-s_{1}^{2}\left(\xi^{h}\right) s_{2}^{2}\left(\xi^{h}\right), \\
& \phi^{d}=\phi^{2}=q+\alpha p-c\left(\xi^{h}, \xi^{s}\right), \\
& \phi^{v}=\phi^{3}=q-c_{v}\left(\xi^{h}\right),
\end{aligned}
$$

where $p=\frac{1}{3} \operatorname{tr} \tau$ and $q=\sqrt{\operatorname{dev} \tau: \operatorname{dev} \tau}$. The relationships between the constitutive parameters $s_{1}$, $s_{2}$ and $c_{v}$, and the internal variable $\xi^{h}$, are given by the following (highly non-linear) hardening laws:

$$
\begin{aligned}
s_{1}\left(\xi^{h}\right) & =\frac{\eta_{0}}{\xi^{h}} A_{s_{1}}\left(\ln \left(\frac{1-\eta_{\mathrm{app}}}{1-\xi^{h}}\right)\right)^{\frac{1}{N_{s_{1}}}}, \\
s_{2} & =\sqrt{\frac{3\left(1-k_{\mathrm{tr}}\right)}{1+2 k_{\mathrm{tr}}}} \text { with } \\
k_{\mathrm{tr}}\left(\xi^{h}\right) & = \begin{cases}k_{\mathrm{tr} 0} & \text { if } \xi^{h} \leqslant \eta_{k_{\mathrm{tr}}}, \\
\left(\xi^{h}-\eta_{k_{\mathrm{tr}}}\right)\left(A_{k_{\mathrm{tr}}} \xi^{h}+B_{k_{\mathrm{tr}}}\right)+k_{\mathrm{tr} 0} & \text { if } \xi^{h}>\eta_{k_{\mathrm{tr}}},\end{cases}
\end{aligned}
$$

\footnotetext{
$\overline{\text { \$The factor } \eta_{0} / \xi^{h}}$ appears to put into correspondence true stress data with the Kirchhoff stress values. 


$$
c_{v}\left(\xi^{h}\right)= \begin{cases}q_{\text {int }} & \text { if } \xi^{h} \leqslant \xi_{v 0}, \\ \sqrt{\frac{2}{3}} \frac{\eta_{0}}{\xi^{h}} \sigma_{y}\left(1-A_{c_{v \mathrm{~h}}}\left(\xi^{h}-B_{c_{v \mathrm{~h}}}\right)\left(1-\xi^{h}\right)\right) & \text { if } \xi^{h}>\xi_{v 0} .\end{cases}
$$

where $\eta_{\text {app }}$ stands for the relative apparent density, defined as the ratio of the apparent density $\rho_{\text {app }}$ to the theoretical density $\rho_{\text {th }}$ of the corresponding metal or alloy. The fitted constant for a Distaloy AE alloy with $\rho_{\text {app }}=3.04 \mathrm{~g} / \mathrm{cm}^{3}$ and $\rho_{\text {th }}=7.33 \mathrm{~g} / \mathrm{cm}^{3}$ are: $A_{s_{1}}=95.4439 \mathrm{MPa}$ and $N_{s_{1}}=0.6506$, for (9); $A_{k_{\mathrm{tr}}}=1.074, B_{k_{\mathrm{tr}}}=0.242, k_{\mathrm{tr} 0}=1 / 3$ and $\eta_{k_{\mathrm{tr}}}=0.635$, for $(10)$; and $A_{c_{v \mathrm{~h}}}=58.160, B_{c_{v \mathrm{~h}}}=$ $0.8252, \sigma_{y}=370 \mathrm{MPa}$ and $\xi_{v 0}=0.92$, for (11). The variable $q_{\text {int }}$ is the deviatoric stress measure at the intersection between the Drucker-Prager and elliptical cap $\left(\partial \mathbb{E}_{\tau}^{d, e}\right)$. The first part of (11) indicates, thus, that for $\xi^{h}<\xi_{v 0}$, the Von Mises yield surface reduces to a point, the intersection between the other two yield surfaces, and comes actually into play only for $\xi^{h}>\xi_{v 0}$ (high levels of compaction, see Figure 1).

In (7), the parameter $\alpha$ is considered constant $(\alpha=2.29)$. The variable $c$, on the other hand, is customarily referred to as cohesion and can be interpreted as the shear strength under zero hydrostatic stress (intersection of the Drucker-Prager yield surface with the $q$-axis); it is the only state variable of the problem that depends on both internal variables $\xi^{h}$ and $\xi^{s}$. We further assume that the following decomposition for the rate of change of cohesion holds:

$$
\dot{c}=\dot{c}_{h}+\dot{c}_{S},
$$

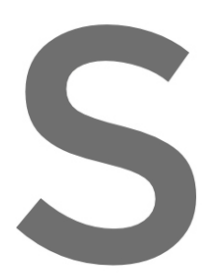

where the subscripts $h$ and $s$ denote hardening and softening, respectively. The hardening law $c_{\mathrm{h}}$
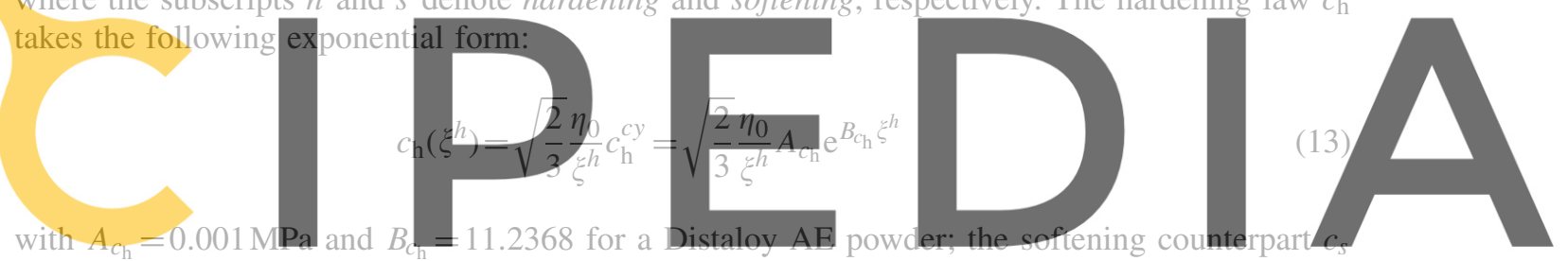

displays also an exponential format

\section{Register for free at https//www.scipedia.com to download the version without the watermark $c_{S}\left(\xi^{h}, \xi^{s}\right)=c_{0}\left(\exp \left(\frac{10 \zeta}{c_{0}}\right)-1\right)$,

where $H_{0}$ denotes the softening parameter and $c_{0}$ stands for the cohesion at the moment $t_{i s}$ in which yielding on the Drucker-Prager yield surface commences, that is, $c_{0}=c_{\mathrm{h}}\left(\xi^{h}\left(t_{i s}\right)\right)$. The tangential

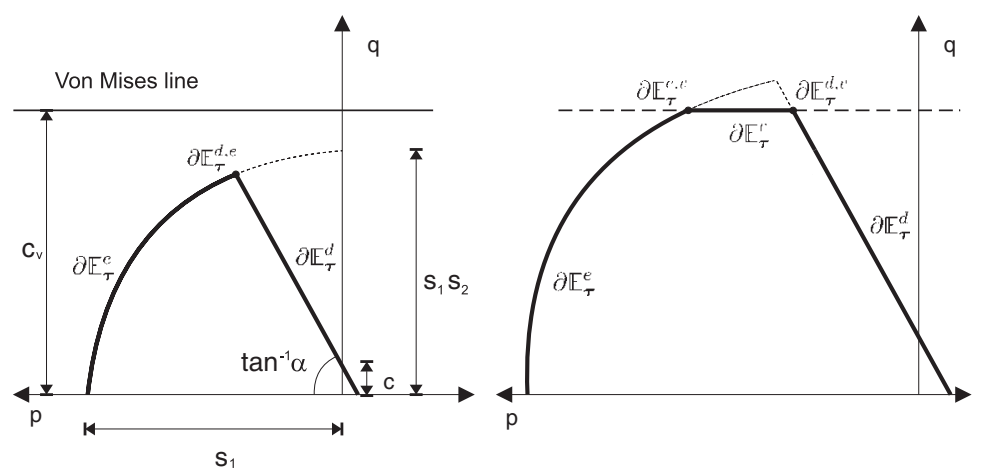

(a)

(b)

Figure 1. Yield surfaces for two different states: (a) Drucker-Prager + elliptical cap, for moderate level of compaction and (b) Drucker-Prager + elliptical cap + Von Mises, for high level of compaction (close to the theoretical density of the fully dense material). 
slope $H$ of the curve described by (14) is termed the continuum softening modulus [22], and it can be expressed as

$$
H=H_{0} \frac{c_{0}+c_{s}}{c_{0}}=H_{0} \frac{c}{c_{0}} .
$$

To alleviate the acclaimed shortcoming of the lack of convergence upon refinement of the finite element mesh, typical of softening models, the softening modulus is related to the localization bandwidth $l_{f}$ through the expression $H_{0}=l_{f} \hat{H}_{0}$ [23]. The so-called intrinsic softening parameter [22], denoted as $\hat{H}_{0}$, is calibrated from experimentally obtained fracture energy values; as mentioned earlier, the toughness of the powder increases with the level of densification $\left(\xi^{h}\right)$, and this consideration is reflected in the following empirical correlation for $\hat{H}_{0}$ :

$$
\left|\hat{H}_{0}\left(\xi^{h}\right)\right|=\frac{\eta_{0}}{\xi^{h}} A_{H} \exp \left(B_{H} \xi^{h}\right),
$$

where $A_{H}=3.574 \mathrm{~N} / \mathrm{mm}^{3}$ and $B_{H}=12.963$.

\subsection{Flow rules and evolutionary equations for the internal variables}

Koiter's generalization of the flow rule is applied to obtain the rate of plastic deformation tensor $\mathbf{d}_{\mathrm{p}}$ in terms of the plastic flow vectors $m^{\alpha}$ associated with each yield surface
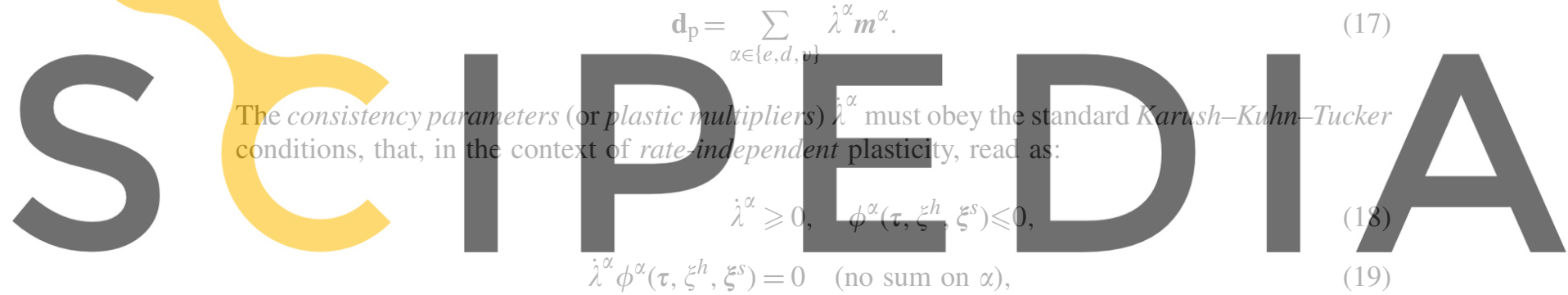

(19)

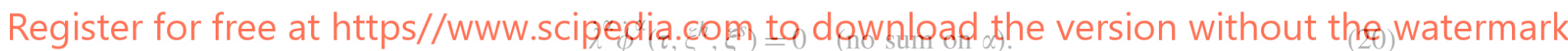

Flow rules are associative on both the elliptical and the Von Mises yield surface

$$
\begin{aligned}
& \boldsymbol{m}^{e}=\frac{\partial \phi^{e}}{\partial \tau}=2 \operatorname{dev} \tau+\frac{2}{3} s_{2}^{2} p \mathbf{1} \\
& \boldsymbol{m}^{v}=2 \operatorname{dev} \tau
\end{aligned}
$$

and non-associative on the Drucker-Prager envelope, on which the plastic potential function $Q^{d}$ defines a parabola in the mean-deviatoric stress plane

$$
Q^{d}=q^{2}+\gamma c \alpha p \rightarrow \boldsymbol{m}^{d}=\frac{\partial Q^{d}}{\partial \tau}=2 \operatorname{dev} \tau+\frac{1}{3} \gamma c \alpha \mathbf{1},
$$

where $\gamma$ is a dilatancy constant close to zero ( typically $\gamma=0.01$; for $\gamma=0$, the isochoric flow rule is recovered).

One of the fundamental assumptions on which the constitutive model under consideration is built is that the magnitude of elastic strains is small in comparison with irrecoverable deformations. This assumption affords to draw an approximation between $\xi^{h}$ (accumulated compressive plastic strains) and the relative density $\eta$. Accordingly, the evolution equation for the internal hardening variable can be written as

$$
\dot{\xi}^{h}=\xi^{h} \mathscr{H}(\dot{\eta}) \mathscr{H}\left(\dot{\lambda}^{e}\right) \frac{\dot{\eta}}{\eta}=\mathscr{H}\left(\dot{\lambda}^{e} \dot{\eta}\right) \frac{\dot{\eta}}{\eta}
$$


where $\mathscr{H}(\bullet)$ denotes the Heavyside step function. The factor $\mathscr{H}(\dot{\eta})$ in the above equation accounts for the fact that $\xi^{h}$ can only evolve if $\dot{\eta}>0$, i.e. if density increases at the analyzed point. The other Heaviside factor, $\mathscr{H}\left(\dot{\lambda}^{e}\right)$, adds the proviso that the elliptical yield surface must be active $\left(\dot{\lambda}^{e}>0\right)$ for $\xi^{h}$ to increase. The major benefit arising from this approximation is that, once the sign of $\dot{\lambda}^{e}$ is known, the differential equation represented by expression (24) can be integrated in closed form.

Finally, the evolutionary equation for the internal softening variable takes the following form:

$$
\xi^{s}=2\left(1-\mathscr{H}\left(\dot{\lambda}^{e}\right)\right) \dot{\lambda}^{d} q
$$

In virtue of the above equation, the internal softening variable only evolves when the stress state is on the Drucker-Prager yield surface. The Heaviside term in the above equation precludes the possibility of both internal variables increasing simultaneously at the intersection of the DruckerPrager and the elliptical yield surface.

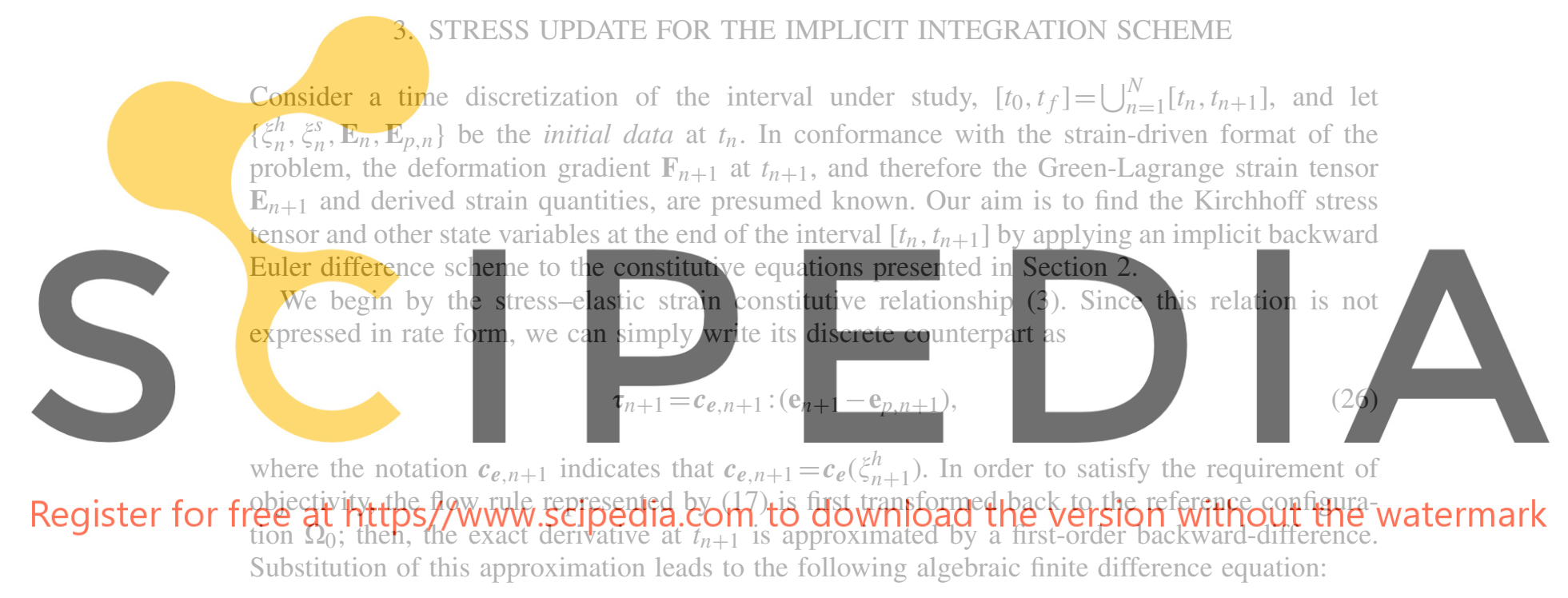

$$
\mathbf{E}_{p, n+1}=\mathbf{E}_{p, n}+\sum_{\beta \in \unlhd_{n+1}^{\text {act }}} \Delta \lambda_{n+1}^{\beta} \varphi^{*}\left(\boldsymbol{m}_{n+1}^{\beta}\right),
$$

where $\Delta \lambda_{n+1}^{\beta}$ denotes the discrete plastic multiplier $\Delta \lambda_{n+1}^{\beta}=\Delta t_{n+1} \dot{\lambda}^{\beta}$, with $\Delta t_{n+1}=t_{n+1}-t_{n} ; \rrbracket_{n+1}^{\text {act }}$ is the set of active constrains, defined as $\sqrt{n+1}_{n+1}^{\text {act }}=\left\{\beta \in\{1,2,3\} \mid \Delta \lambda_{n+1}^{\beta}>0\right\}$; and the symbol $\varphi^{*}(\bullet)$ stands for the pull-back operator ${ }^{\S} \varphi^{*}(\bullet)=\mathbf{F}_{n+1}^{T} \cdot(\bullet) \cdot \mathbf{F}_{n+1}$. We revert again to the spatial description by obtaining the push forward, defined as $\varphi_{*}(\bullet)=\mathbf{F}_{n+1}^{-T} \cdot(\bullet) \cdot \mathbf{F}_{n+1}^{-1}$, of (27)

$$
\begin{aligned}
\varphi_{*}\left(\mathbf{E}_{p, n+1}\right) & =\varphi_{*}\left(\mathbf{E}_{p, n}\right)+\sum_{\beta \in \unlhd_{n+1}^{\text {act }}} \Delta \lambda_{n+1}^{\beta} \varphi_{*}\left(\varphi^{*}\left(\boldsymbol{m}_{n+1}^{\beta}\right)\right) \Rightarrow \\
\mathbf{e}_{p, n+1} & =\breve{\mathbf{e}}_{p, n}+\sum_{\beta \in J_{n+1}^{\text {act }}} \Delta \lambda_{n+1}^{\beta} \boldsymbol{m}_{n+1}^{\beta},
\end{aligned}
$$

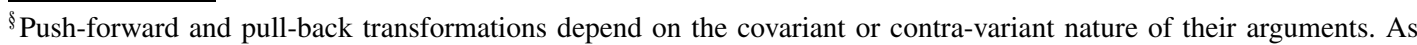
usual, strain measures are considered here covariant tensors, whereas stress quantities are regarded as contravariant quantities.
} 
where the 'breve' symbol in $\breve{\mathbf{e}}_{p, n}$ is attached to indicate that $\breve{\mathbf{e}}_{p, n}$ is a strain measure computed at time $t_{n}$ but transformed to the current configuration $\Omega_{n+1}$ via a push-forward operation, i.e.

$$
\breve{\mathbf{e}}_{p, n}=\varphi_{*}\left(\mathbf{E}_{p, n}\right)=\mathbf{F}_{n+1}^{-T} \cdot \mathbf{E}_{p, n} \cdot \mathbf{F}_{n+1}^{-1}=\Delta \mathbf{F}_{n+1}^{-T} \cdot \mathbf{e}_{p, n} \cdot \Delta \mathbf{F}_{n+1}^{-1} .
$$

In the above, $\Delta \mathbf{F}_{n+1}^{-T}$ denotes the incremental deformation gradient $\Delta \mathbf{F}_{n+1}=\mathbf{F}_{n+1} \cdot \mathbf{F}_{n}^{-1}$.

The same approximation of the time derivative is carried out on the evolutionary equation (25) for the internal softening variable, yielding the following difference equation:

$$
\xi_{n+1}^{s}=\xi_{n}^{s}+2\left(1-\mathscr{H}\left(\Delta \lambda_{n+1}^{e}\right)\right) \Delta \lambda_{n+1}^{d} q_{n+1},
$$

where $q_{n+1}=\left\|\operatorname{dev} \tau_{n+1}\right\|$. As regards the rate equation (24) for the internal hardening variable, it proves more accurate to first express (24) in terms of logarithms and then introduce the approximation of the time derivatives:

$$
\frac{\partial}{\partial t}\left(\log \xi^{h}\right)=\mathscr{H}\left(\dot{\lambda}^{e} \dot{\eta}\right) \frac{\partial}{\partial t}(\log \eta) \Rightarrow \frac{\xi_{n+1}^{h}}{\xi_{n}^{h}}=\left(\frac{\eta_{n+1}}{\eta_{n}}\right)^{\mathscr{H}\left(\Delta \lambda_{n+1}^{e}\left(\eta_{n+1}-\eta_{n}\right)\right)} .
$$

The parameters governing both the size and shape of the elliptical cap surface, and the location of the Von Mises yield surface, can be obtained through simple functional evaluations, i.e. $s_{1, n+1}=$ $s_{1}\left(\xi_{n+1}^{h}\right), s_{2, n+1}=s_{2}\left(\xi_{n+1}^{h}\right)$ and $c_{v, n+1}=c_{v}\left(\xi_{n+1}^{h}\right)$. The cohesion variable $c$ depends on both $\xi^{h}$ and $\xi^{s}$ (see Equations (13) and (14)); its update formula is derived thus from approximating the derivative of the cohesion rate equation
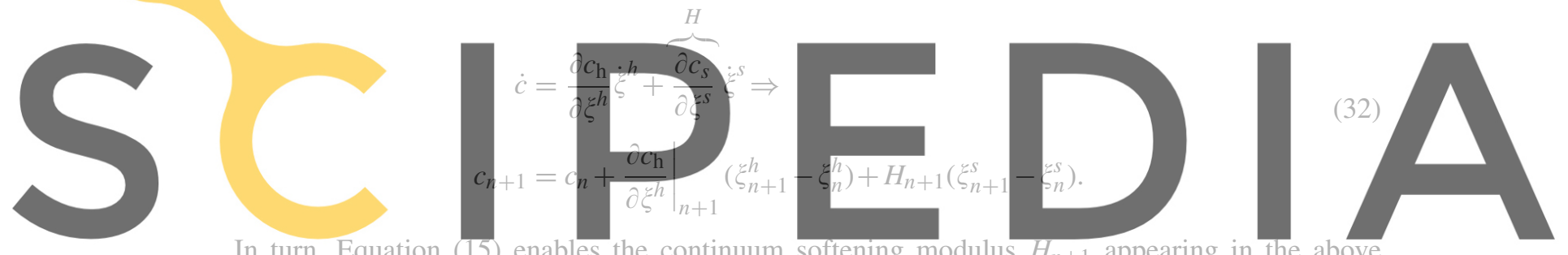

In turn, Equation (15) enables the continuum softening modulus $H_{n+1}$ appearing in the above

equation to be evaluated as

Register for free at https//www.scipedia.com to download the version without the watermark

$$
H_{n+1}=H_{0}\left(\xi_{n+1}^{h}\right) \frac{c_{n+1}}{c_{0}\left(\xi_{n+1}^{h}\right)} \text {. }
$$

The above equation can be simplified if we take into account that, according to Equation (25), the simultaneous increase of both internal variables is not possible. Since the internal softening variable $\xi^{s}$, and therefore also $H_{n+1}$, only evolves when the Drucker-Prager yield surface is active, it follows that, in this situation, $\xi_{n+1}^{h}=\xi_{n}^{h}$ and $c_{0}\left(\xi_{n+1}^{h}\right)=c_{\mathrm{h}}\left(\xi_{n}^{h}\right)=c_{\mathrm{h}, n}$; thus, (33) can be rewritten as

$$
H_{n+1}=H_{0, n} \frac{c_{n+1}}{c_{\mathrm{h}, n}} \text {. }
$$

Finally, the discrete counterpart of the Karush-Kuhn-Tucker loading/unloading conditions can be written as

$$
\begin{gathered}
\Delta \lambda_{n+1}^{\beta} \geqslant 0, \phi_{n+1}^{\beta} \leqslant 0 \quad(\beta=1,2,3), \\
\Delta \lambda_{n+1}^{\beta} \phi_{n+1}^{\beta}=0(\beta=1,2,3) \quad(\text { no sum on } \beta),
\end{gathered}
$$

where

$$
\begin{aligned}
& \phi_{n+1}^{e} \equiv \phi_{n+1}^{1}=q_{n+1}^{2}+s_{2, n+1}^{2} p_{n+1}^{2}-s_{1, n+1}{ }^{2} s_{2, n+1}{ }^{2}, \\
& \phi_{n+1}^{d} \equiv \phi_{n+1}^{2}=q_{n+1}+\alpha p_{n+1}-c_{n+1}, \\
& \phi_{n+1}^{v} \equiv \phi_{n+1}^{3}=q_{n+1}-c_{v, n+1} .
\end{aligned}
$$


To cast the loading-unloading conditions in a format readily amenable to computational implementation, we introduce the notion of trial elastic state (see e.g. [24]), which arises from assuming elastic behavior throughout the time step $\left[t_{n}, t_{n+1}\right]$. Accordingly, the 'trial' counterparts at $t_{n+1}$ of the plastic Almansi strain tensor, the internal variables and the Kirchhoff stress tensor are expressible as

$$
\begin{gathered}
\mathbf{e}_{p, n+1}^{\operatorname{tr}}=\breve{\mathbf{e}}_{p, n}, \\
\xi_{n+1}^{h, t r}=\xi_{n}^{h}, \\
\xi_{n+1}^{s, t r}=\xi_{n}^{s}
\end{gathered}
$$

and

$$
\tau_{n+1}^{\mathrm{tr}}=\boldsymbol{c}_{\boldsymbol{e}, n}:\left(\mathbf{e}_{n+1}-\breve{\mathbf{e}}_{p, n}\right)
$$

respectively. If the trial stress defined in (43) lies within the elastic domain determined by the prevailing yield condition, the incremental deformation is entirely elastic, and hence $\tau_{n+1}=\tau_{n+1}^{\mathrm{tr}}$. By contrast, if the yield condition is violated, that is, if

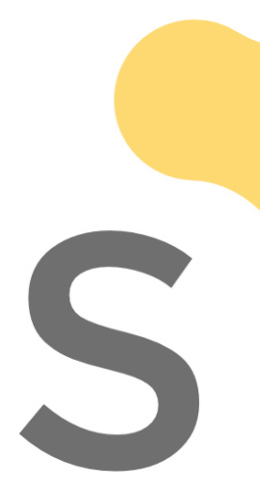

$$
\phi_{n+1}^{\beta, \operatorname{tr}}>0 \text { for any } \beta=1,2,3,
$$

the tentative assumption of elastic response is rejected, and the updated state is obtained by 'returning' the trial state to the yield surface. To determine the 'return' direction, it proves convenient to combine Equations (26) and (28) with the preceding definition of trial stress. An easy manipulation of these expressions leads to the following result:

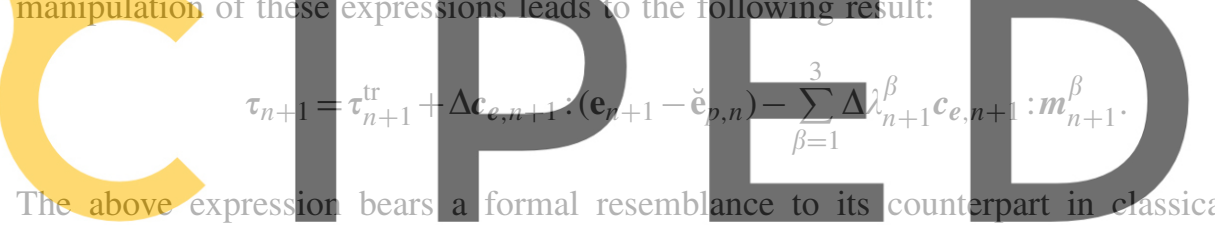

plasticity (see e.g. [24]), a fact attributable to the assumption of negligible elastic strains. Apart Register for frem the plastic strain tensor at $t_{n} \breve{p}$, which is affected by the incremental push-foryard operator,

this fourth-order tensor is defined as

$$
\Delta \boldsymbol{c}_{\boldsymbol{e}, n+1}=\boldsymbol{c}_{\boldsymbol{e}}\left(\xi_{n+1}^{h}\right)-\boldsymbol{c}_{\boldsymbol{e}}\left(\xi_{n}^{h}\right)=\left(\kappa_{n+1}^{e}-\kappa_{n}^{e}\right) \mathbf{1} \otimes \mathbf{1}+2\left(\mu_{n+1}^{e}-\mu_{n}^{e}\right) \boldsymbol{I}_{\mathrm{dev}}
$$

and reveals the coupling between elastic and plastic responses, i.e. the fact that the elastic moduli $E^{e}$ increases as hardening progresses.

\subsection{Fractional step-based return-mapping algorithm}

It was pointed out in the introductory section that the presence of softening and the highly non-linear, tightly coupled nature of the problem affects adversely the solution of the above return-mapping equations by means of standard procedures. The peculiar feature of the proposed return-mapping algorithm is the iterative process adopted to 'return' the trial stress to the yield surface, which is not a standard Newton-Raphson scheme or variants thereof; rather, it has the flavor of fractional step methods (FSM), since it is based on the decoupling of the evolution equations for the plastic strains and the internal variables. It can be viewed also as a natural extension of the two-step algorithm derived from the elastic-plastic operator split, in the sense that it involves the repetitive application of a predictor-corrector procedure. The steps of the algorithm are thoroughly detailed in Box 3.1. In each iteration, the predictor state arises from freezing the internal variables and solving the resulting return-mapping problem (Equations (49) and (50)); in the corrector step, the update of the internal variables (Equations (52) and (53)) using the value of the stress state computed in the predictor stage is performed. The cycle is repeated until a convergence criterion is met. 
1. Compute trial elastic stress

$$
\begin{aligned}
\tau_{n+1}^{\mathrm{tr}} & =\boldsymbol{c}_{\boldsymbol{e}, n}:\left(\mathbf{e}_{n+1}-\breve{\mathbf{e}}_{p, n}\right), \\
\rrbracket_{n+1}^{\mathrm{act}, \mathrm{tr}} & =\left\{\beta \in\{e, d, v\} \mid \phi_{n+1}^{\beta, \operatorname{tr}}>0\right\} .
\end{aligned}
$$

IF $\rrbracket_{n+1}^{\text {act, tr }}=\emptyset$ THEN

ELSE

$$
\text { Set }(\bullet)_{n+1}=(\bullet)_{n+1}^{* t r} \text { EXIT }
$$

ENDIF

$$
\mathrm{k}=1, \rrbracket^{\mathrm{act},(1)}=\sqrt{n+1}_{n+1}^{\mathrm{act}, \mathrm{tr}}, \xi^{(0)}=\left[\xi_{n}^{h}, \xi_{n}^{s}\right]
$$

2. Predictor step of the FSM return-mapping algorithm. Obtain $\tau^{(k)}$ and $\Delta \lambda^{\beta,(k)}, \beta \in \mathbb{ل}^{\text {act, }(k)}$ solving*

$$
\boldsymbol{\tau}^{(k)}=\tau_{n+1}^{\mathrm{tr}}+\Delta \boldsymbol{c}_{\boldsymbol{e}}^{(k-1)}:\left(\mathbf{e}_{n+1}-\breve{\mathbf{e}}_{p, n}\right)-\sum_{\beta \in J^{\mathrm{Jct},(k)}} \Delta \lambda^{\beta,(k)} \boldsymbol{c}_{\boldsymbol{e}}^{(k-1)}: \boldsymbol{m}^{\beta,(k)}, \phi^{\beta}\left(\boldsymbol{\tau}^{(k)}, \boldsymbol{\xi}^{(k-1)}\right)
$$

$$
\forall \beta \in J^{\text {act, }(k)},
$$

where

$$
\boldsymbol{c}_{\boldsymbol{e}}^{(k-1)}=\boldsymbol{c}_{\boldsymbol{e}}\left(\boldsymbol{\xi}^{(k-1)}\right), \quad \boldsymbol{m}^{\beta,(k)}=\boldsymbol{m}^{\beta}\left(\tau^{(k)}, \boldsymbol{\xi}^{(k-1)}\right)
$$

(* If the active set $\rrbracket^{\text {act, }(k)}$ contains more than two indices, this system has to be solved for each pair of indices).

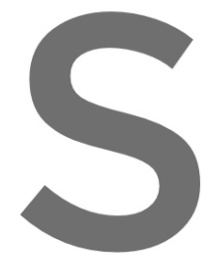

IF $\Delta \lambda$

Reset $₫$

Goto to

ENDIF

3. Corrector step. Update of the internal variables.
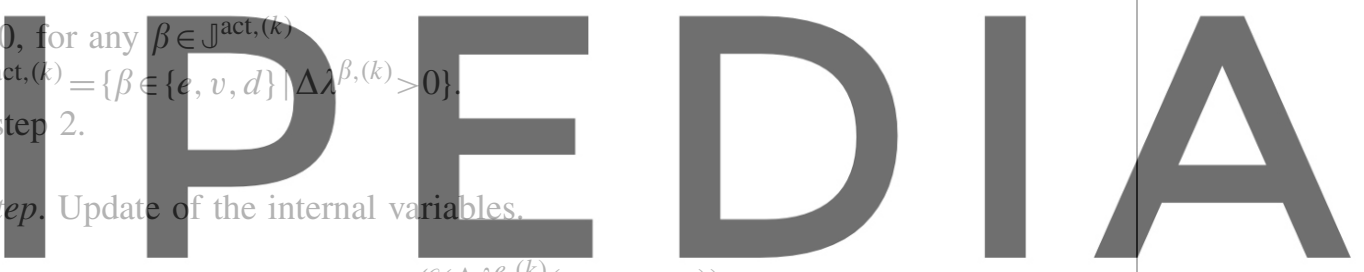

Register for free at https//www.scipedial.com to download the version without the

$$
\xi^{s,(k)}=\xi_{n}^{s}+2\left(1-\mathscr{H}\left(\Delta \lambda^{e,(k)}\right)\right) \Delta \lambda^{d}\left(\tau^{(k)}, \xi^{(k)}\right) q^{(k)} .
$$

4. Check convergence

$\mathrm{IF}\left|\phi^{\beta}\left(\tau^{(k)}, \boldsymbol{\xi}^{(k)}\right)\right|<T O L_{1} \quad \forall \beta \in \mathbb{ل}^{\mathrm{act},(k)}$ AND $\left\|\boldsymbol{\xi}^{(k)}-\boldsymbol{\xi}^{(k-1)}\right\|<T O L_{2}$

Set $(\bullet)_{n+1}=(\bullet)_{k}$ EXIT

ELSE

Set $k \leftarrow k+1$. Goto step 2 .

ENDIF

Box 3.1: Basic steps of FMS return-mapping algorithm.

3.1.1. Predictor stage. Keeping $\xi^{h}$ and $\xi^{s}$ fixed amounts to assume that the yield surfaces remain unchanged in stress space during this predictor step; the problem can be, thus, legitimately regarded as a perfect plasticity return-mapping problem. The question of the existence and uniqueness of solution in the predictor stage is easily resolved, since, in a perfect plasticity case, a sufficient condition for existence and uniqueness is the convexity of the elastic domain in stress space ${ }^{\mathbb{I l}}$ [24]. The computational effort required to obtain this unique solution varies depending on the active

"Examination of Figure 1, along with straightforward geometric considerations, permits to conclude that the elastic domain enclosed by the considered yield surfaces is indeed convex in stress space. 
constraints at each iteration; however, as we argue in the following discussion, it can be computed in closed form, regardless of the yield surface (or surfaces) the trial stress is projected onto.

Elliptical cap: The elliptical yield surface (with associative flow rule) falls within the class of general quadratic models of classical plasticity, wherein the solution of the corresponding returnmapping equations can be computed by solving a quartic equation [24]. To derive such equation, first, (49) is particularized to the case in which only the elliptical cap is active, and then resolved into its mean and deviatoric components $\|$

$$
\begin{aligned}
p^{(k)} & =\frac{p_{u p}^{(k-1)}}{1+2 \kappa^{e,(k-1)} \Delta \lambda^{e,(k)} s_{2}^{(k-1)^{2}},} \\
q^{(k)} & =\frac{q_{u p}^{(k-1)}}{1+4 \mu^{e,(k-1)} \Delta \lambda^{e,(k)}},
\end{aligned}
$$

where

$$
\begin{aligned}
& p_{u p}^{(k-1)}=p_{n+1}^{\mathrm{tr}}+\Delta \kappa^{e,(k-1)} \operatorname{tr}\left(\mathbf{e}_{n+1}-\breve{\mathbf{e}}_{p, n}\right), \\
& q_{u p}^{(k-1)}=\left\|\operatorname{dev} \tau_{n+1}^{\mathrm{tr}}+2 \Delta \mu^{e,(k-1)} \operatorname{dev}\left(\mathbf{e}_{n+1}-\breve{\mathbf{e}}_{p, n}\right)\right\| .
\end{aligned}
$$

After substituting Equations (54) and (55) into the yield condition (37), we arrive at the following quartic polynomial in $q^{(k)}$ :

$$
a_{4} q^{4}+a_{3} q^{3}+a_{2} q^{2}+a_{1} q+a_{0}=0
$$

where the coefficients $a_{i}, i=1, \ldots, 4$ are given by

$$
\begin{aligned}
& \left.a_{4}=b_{2}^{2}, \quad a_{3}=2 b_{1} s_{2}^{2} q_{u p} b_{2}, \quad a_{2}=s_{2}^{2}\left(\left(q_{u p} s_{2} b_{1}\right)^{2}+p_{u p}^{2}-s_{1}^{2} b_{2}^{2}\right)\right), \\
& a_{1}=-2 s_{2}^{2}\left(s_{1} s_{2}\right)^{2} b_{1} q_{u p} b_{2}, \quad a_{0}=-\left(s_{1} s_{2}^{3} b_{1} q_{u p}\right)^{2}
\end{aligned}
$$

and

$$
b_{2}=\left(1-b_{1} s_{2}^{2}\right), \quad b_{1}=\frac{\kappa^{e}}{2 \mu^{e}} .
$$

The unique positive root of Equation (58) in the interval $\left[0, s_{1} s_{2}\right]$ can be determined in closed form by a modified version, proposed by Simo [24], of the classical solution procedure for quartic equations. Once the values of $p^{(k)}$ and $q^{(k)}$ have been obtained, the updated stress tensor can be calculated as

$$
\tau^{(k)}=p^{(k)} \mathbf{1}+\frac{q^{(k)}}{q_{u p}^{(k-1)}}\left(\operatorname{dev} \tau_{n+1}^{\mathrm{tr}}+2 \Delta \mu^{e,(k-1)} \operatorname{dev}\left(\mathbf{e}_{n+1}-\breve{\mathbf{e}}_{p, n}\right)\right) .
$$

Drucker-Prager and Von Mises yield surfaces: The 'predictor' stress when either the Von Mises or the Drucker-Prager yield surfaces can be calculated also in closed form. In the case of the Von Mises yield surface, the deviatoric nature of the plastic flow on such surface renders such a task simple matter: $q^{(k)}=c_{v}^{(k-1)}$ and $p^{(k)}=p^{(k-1)}$. The computation of the updated stress when only the Drucker-Prager yield surface is active is also trivial; it only involves the solution of a quadratic polynomial in $q^{(k-1)}$. The expression of this polynomial is derived following the same logic leading to (58):

$$
\Gamma\left(q^{(k)}\right)=q^{(k)^{2}}+\left(\alpha p_{n+1}^{\mathrm{tr}}+c^{(k-1)}(\chi-1)\right) q^{(k)}-\chi c^{(k-1)} q_{n+1}^{\mathrm{tr}}=0,
$$

\footnotetext{
${ }^{\|}$For notational simplicity, we omit the index $n+1$ to those variables that are updated during the iterative process, i.e. $\tau^{(k)}=\tau^{n+1,(k)}$.
} 
where

$$
\chi=\alpha^{2} \gamma \frac{\kappa^{e}}{4 \mu^{e}}
$$

Besides, it can be easily deduced, by virtue of the intermediate value theorem, that the desired root must lie in the interval $\left[0, q_{n+1}^{\mathrm{tr}}\right]$.

Corner points: In situations in which the set of active constraints $\rrbracket^{\text {act, }(k)}$ contains two indices, the enforcement of the plastic consistency conditions places the stress $\tau^{(k)}$ at the interception point of the corresponding active yield surfaces. As the yield condition is constructed by a quadratic function (the elliptical cap yield surface $\partial \mathbb{E}_{\tau}^{e}$ ) and two affine functions (the Drucker-Prager yield surface $\partial \mathbb{E}_{\tau}^{d}$ and the Von Mises yield surface $\partial \mathbb{E}_{\tau}^{e}$ ), this intersection can be obtained in closed form by solving, at worst, a quadratic equation. Following a common procedure in nonsmooth multisurface plasticity [24], the task of ascertaining whether the two presumably active yield constraints are indeed active is carried out by checking whether the corresponding plastic multipliers are, as KuhnTucker complementary conditions demand, positive. If not, the index associated to the negative plastic multiplier is discarded from the set of active constraints, and the predictor step is performed again with the new set of active constraints.

3.1.2. Corrector stage. The corrector step involves the update of the internal variables (Equations (52) and (53) in Box 3.1) using the stress value $\tau^{(k)}$ computed in the predictor stage.

Internal hardening variable: The evolutionary Equation (52) for the internal hardening variable $\xi^{h}$, however, is solely governed by the change in relative density (which is prescribed during the iterations) and the nonzero character of $\Delta \lambda^{e,(k)}$; the value of $\tau^{(k)}$ is, hence, not actually needed for updating $\xi^{h,(k)}$. This aspect of simplicity, which, we recall, stems from the assumption of negligible elastic strains, can be exploited to increase the speed (per step) of the algorithm. It proves advantageous to, before going through the predictor step, check whether $\eta_{n+1}>\eta_{n}$ and $\Delta \lambda_{n+1}^{e}>0$. Such being the case, the predictor stage in the first iteration can be skipped and one can directly update the internal variable as $\xi^{h,(1)}=\xi_{n}^{h} \eta_{n+1} / \eta_{n}$. Then, the trial stress is projected back to the updated yield surfaces: convergence of the iterative predictor-corrector scheme will be achieved in only one effective iteration.

Internal softening variable: Note that the simultaneous increase, in the same iteration, of both internal hardening and softening variables cannot take place: the purposefully introduced factor $\left(1-\mathscr{H}\left(\Delta \lambda^{e,(k)}\right)\right)$ in $(53)$ precludes this possibility. The internal softening variable, thus, only evolves when plastic yielding occurs on the Drucker-Prager failure envelope. Observe also that the righthand side of the evolutionary equation (53) does depend on the current stress through its deviatoric part and the consistency parameter. An important implication of this is that, in contrast to the situation encountered above, the FSM predictor-corrector scheme cannot be solved exactly in a finite number of iterations; the number of iterations required to achieve convergence will depend on the prescribed error threshold and on the corresponding rate of convergence.

3.1.3. Convergence analysis when the Drucker-Prager envelope is active. To improve the confidence in the proposed FSM return-mapping algorithm, thus, it is crucial to ascertain whether the algorithm is convergent to a unique solution when the Drucker-Prager yield surface is active, and to analyze the conditions under which such a desirable feature, if it exists, can be guaranteed. The strategy adopted in the following to accomplish this task is to reduce the predictor-corrector scheme to a single recursion formula in terms of the norm of the deviatoric stress; then, the convergence characteristics of the sequence $\left\{q^{(k)}\right\}_{k=0}^{\infty}$ defined by this recursion will be analyzed by means of fundamental theorems of calculus.

To this end, the polynomial equation (63) for the predictor stage is first solved for $c^{(k-1)}$ :

$$
\Theta_{p d}=c^{(k-1)}=\frac{q^{(k)}\left(q^{(k)}+\alpha p_{n+1}^{\mathrm{tr}}\right)}{\frac{1}{2} \chi\left(q_{n+1}^{\mathrm{tr}}-q^{(k)}\right)+q^{(k)}} .
$$


A similar relation for the corrector equation (53) can be drawn; from the deviatoric component of (49), one can deduce first the value of the plastic multiplier $\Delta \lambda^{d,(k)}$ as a function of $q$

$$
\Delta \lambda^{d,(k)}=\frac{1}{4 \mu_{n}^{e}} \frac{q_{n+1}^{\mathrm{tr}}-q^{(k)}}{q^{(k)}} .
$$

Upon substitution of this value into Equation (53), we obtain

$$
\xi^{s,(k)}=\xi_{n}^{s}+\frac{q_{n+1}^{\mathrm{tr}}-q^{(k)}}{2 \mu_{n}^{e}} .
$$

Now, inserting the above equation into the discrete version of the softening law (Equation (32)), and making use of the definition of softening modulus (34), we find that

$$
c^{(k)}=c_{n}+\frac{\hat{H}_{0, n}}{c_{\mathrm{h}, n}} 2 \mu_{n}^{e} c^{(k)}\left(q_{n+1}^{\mathrm{tr}}-q^{(k)}\right),
$$

where $\hat{H}_{0, n}=H_{0, n} /\left(2 \mu_{n}^{e}\right)$. Solving Equation (68) for $c^{(k)}$, we obtain the counterpart of Equation (65) for the corrector stage:

$$
\Theta_{c r}=c^{(k)}=\frac{c_{n}}{1-\frac{\hat{H}_{0, n}}{c_{\mathrm{h}, n}}\left(q_{n+1}^{\mathrm{tr}}-q^{(k)}\right)}=\frac{c_{n}}{1+\frac{\left|\hat{H}_{0, n}\right|}{c_{\mathrm{h}, n}}\left(q_{n+1}^{\mathrm{tr}}-q^{(k)}\right)} .
$$

Finally, the expected recursion formula arises from simply equating the right-hand sides of Equation (65) (for $k \rightarrow k+1$ ) and Equation (69)

$$
\Theta_{p d}\left(q^{(k+1)}\right)=\Theta_{c r}\left(q^{(k)}\right)
$$

that is,

$$
\frac{\overbrace{\frac{q^{(k+1)}\left(q^{(k+1)}+\alpha p_{n+1}^{\mathrm{tr}}\right)}{\Theta_{p d}\left(q^{(k+1)}\right)}}^{\frac{1}{2} \chi\left(q_{n+1}^{\mathrm{tr}}-q^{(k+1)}\right)+q^{(k+1)}}=\frac{\Theta_{c r}\left(q^{(k)}\right)}{c_{n}}}{1+\frac{\left|\hat{H}_{0, n}\right|}{c_{\mathrm{h}, n}}\left(q_{n+1}^{\mathrm{tr}}-q^{(k)}\right)}
$$

with $q^{(0)}=q_{n+1}^{\text {tr }}$.

The above recursion possesses an interesting geometrical interpretation, illustrated in Figure 2. The predictor and corrector equations $\Theta_{p d}=0$ and $\Theta_{c r}=0$ are represented in the $c-q$ plane. The method bears some resemblance to the so-called fixed-point iteration method [25, 26]: the predictor step consists of finding the intersection between the horizontal lines ( $c$ constant) and the predictor curve $\Theta_{p d}=0$; the corrector step, on the other hand involves solving the intersection of the corrector graph $\Theta_{c r}=0$ and the vertical lines ( $q$ constant).

The required conditions for convergence can be intuitively appreciated in Figure 2. Both predictor and corrector curves should be continuous, monotonically increasing functions in the interval $\left[q_{\min }, q_{n+1}^{\mathrm{tr}}\right]$, where $q_{\min }=\max \left(0,-\alpha p_{n+1}^{\mathrm{tr}}\right)^{* *}$; furthermore, they should intersect only at one point. In Appendix A, Proposition A1, the sufficient conditions for the sequence defined by (71) to converge are established in a mathematically formal manner. Moreover, in the same appendix, it is rigorously proved that the particular functional forms of $\Theta_{c r}$ and $\Theta_{p d}$ (Equations (65) and (69), respectively) do satisfy these sufficient conditions. What is more remarkable is that the convergence is unconditional: no matter how far the trial stress may be from the prevailing Drucker-Prager yield surface, or how large may be the values of the softening modulus or other material parameters may be, the method will find invariably the limit of the sequence $\left\{q^{(k)}\right\}_{k=0}^{\infty}$, and hence the updated stress $q_{n+1}$.

\footnotetext{
**From Equation (65) and the positiveness of both $q$ and $c$, it follows that the limit of the sequence must lie in the interval $\left[q_{\mathrm{min}}, q_{n+1}^{\mathrm{tr}}\right]$.
} 


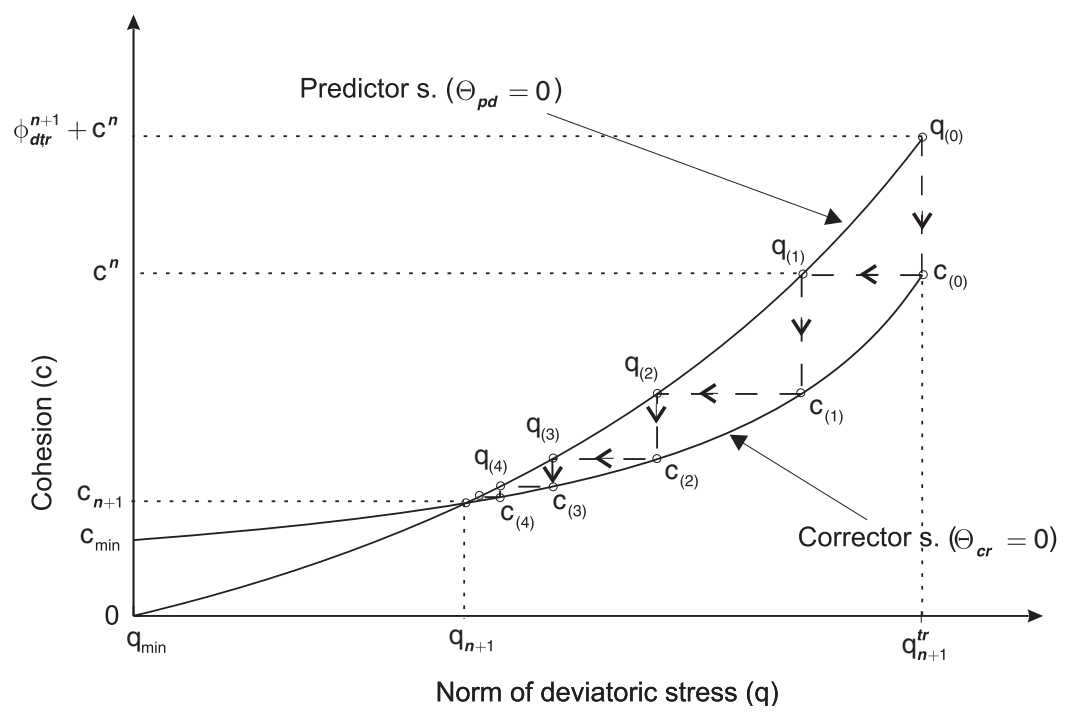

Figure 2. Linearly convergent FSM sequence.

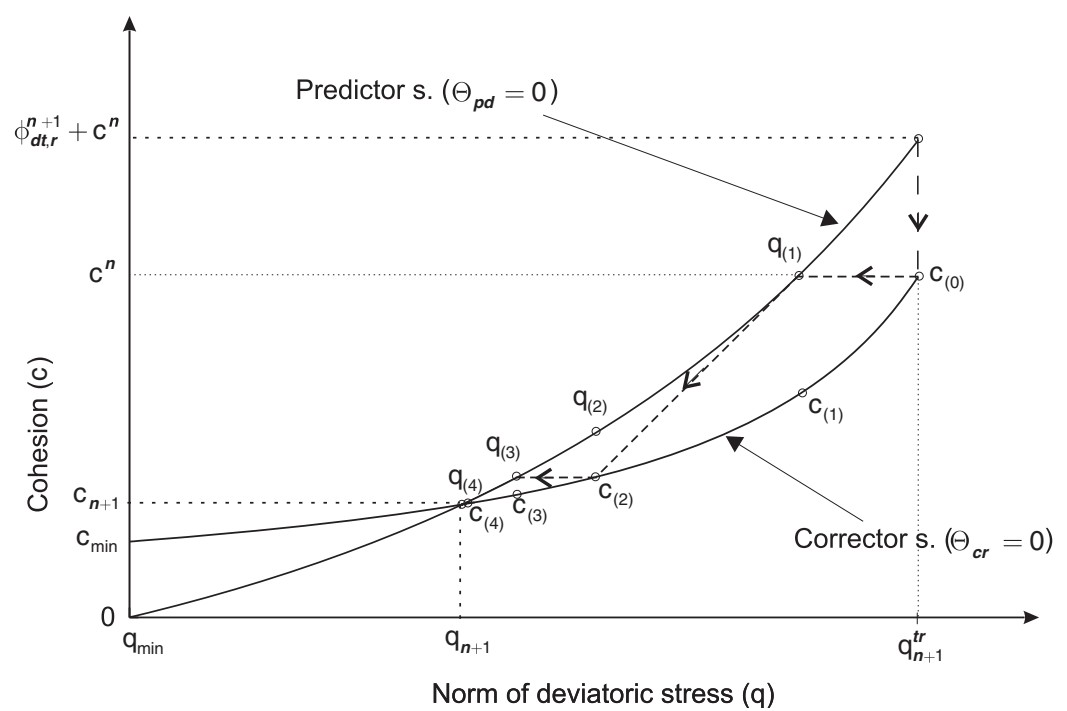

Figure 3. Quadratically convergent FSM sequence.

The issue of rate of convergence of the iterative scheme, on the other hand, is addressed in Proposition A3 of Appendix A. As could be expected-in view of its resemblance to the fixedpoint iterative method-, the rate at which the method approaches the solution is only linear. This relatively slow convergence rate, in comparison with the quadratically Newton-Raphson iterative schemes that accompany other return-mapping algorithm (e.g. closest point projection), raises inevitably the question of how to increase, maintaining the predictor-corrector structure, the rate of convergence of the proposed method.

The plot in Figure 3 illustrates graphically the strategy that can be followed to arrive at a quadratically convergent FSM algorithm: the predictor stage would remain unaltered (projection onto the yield surface with $c$ constant); the corrector step, by contrast, would be modified and accomplished by finding the intersection between the curve $\Theta_{c r}=0$ and the straight line tangent to $\Theta_{p d}=0$ at $q^{(k-1)}$. Observe, however, that this alternative scheme involves the linearization of the predictor function $\Theta_{p d}$, and this exacts a significant price: the convexity of such a function in 
the neighborhood of the solution appears as a necessary condition for convergence. As shown in Appendix A.3, the predictor function is convex whenever the following inequality is satisfied:

$$
\frac{1}{2} \chi \overbrace{\left(q_{n+1}^{\mathrm{tr}}+\alpha p_{n+1}^{\mathrm{tr}}\right)}^{\phi_{n+1}^{d, \mathrm{tr}}}-\alpha p_{n+1}^{\mathrm{tr}}>0 .
$$

Since $\phi_{n+1}^{d, \mathrm{tr}}>0$ and $\chi>0$, it follows that only for $p_{n+1}^{\mathrm{tr}}<0$-when the trial stress falls in the second (compressive) quadrant of the $p-q$ plane - the above inequality holds regardless of the value of the material parameter $\chi=\alpha^{2} \gamma\left(\kappa_{n}^{e} / 4 \mu_{n}^{e}\right)$; for $p_{n+1}^{\text {tr }}>0$, its observation depends on $\chi$ and, thus, the convexity condition cannot be guaranteed for any conceivable trial stress. ${ }^{\dagger \dagger}$ In the interest of robustness, thus, this appealing quadratically convergent FSM scheme is abandoned in favor of the originally proposed linearly convergent one.

\section{IMPL-EX INTEGRATION SCHEME}

We must note emphatically that the stress values computed by the foregoing fully implicit scheme are not actually employed in computing the current internal forces. As pointed out in the preamble, the backward Euler implicit scheme is to be viewed as an auxiliary integration procedure; the information obtained from this scheme at $t_{n}, t_{n-1} \ldots$ is used only at $t_{n+1}$ to update certain variables. The stress values that actually determine the internal forces appearing in the weak formulation of the problem are those furnished by the IMPL-EX integration scheme. This section is devoted to derive the stress update and algorithmic tangent moduli closed-form expressions stemming from this integration procedure.

\subsection{Stress update}

We begin the derivation of the Impl-Ex stress update procedure by selecting those variables to be treated explicitly, i.e. those quantities that do not depend upon the current (at $\left.t_{n+1}\right)$ deformation state. By definition, internal variables, denoted collectively by $\xi$, are monotonically increasing functions of time, i.e. $\dot{\xi} \geqslant 0$; they are logical candidates, thus, to be treated explicitly, since its evolution can be predicted more accurately than other variables exhibiting non-monotonic behavior. To determine the Impl-Ex counterpart of $\xi^{h}$ at $t_{n+1}$, consider, first, Taylor's expansion of the exact solution for the internal variable, denoted by an overbar on the symbol $\xi$, to the governing differential equation at $t_{n+1}$ around $t_{n}$ :

$$
\bar{\xi}_{n+1}=\bar{\xi}_{n}+\left.\frac{\partial \xi}{\partial t}\right|_{\bar{\xi}_{n}} \Delta t_{n+1}+\mathcal{O}\left(\Delta^{2} t_{n+1}\right)
$$

Next, Taylor's expansion is carried out again around $t_{n}$, but evaluated at $t_{n-1}$, yielding

$$
\bar{\xi}_{n}=\bar{\xi}_{n-1}+\left.\frac{\partial \xi}{\partial t}\right|_{\bar{\xi}_{n}} \Delta t_{n}+\mathcal{O}\left(\Delta^{2} t_{n}\right)
$$

If the remainder term in Equation (73) is truncated, the resulting expression would correspond to a explicit finite difference equation. The expression emanating from truncation of the remainder term in Equation (74), on the other hand, is a standard implicit finite difference equation. The application of an explicit difference scheme for marching the solution forward from one time level to the next

\footnotetext{
${ }^{1}$ Interestingly, note that it is precisely because of the lack of convexity-due to the presence of softening - that we refrained from using standard return-mapping algorithms.
} 
time level does not require to solve any system of equation, but presents the inconvenience that, since the yield condition is not enforced at $t_{n+1}$, the error may accumulate and the yield condition may be substantially violated, leading to updated stresses prohibitively far from the yield surface if the time step is not sufficiently small. In implicit difference schemes, by contrast, the plastic consistency condition is enforced at $t_{n+1}$, and hence fulfilled exactly; closed-form expressions for the constitutive tangent operators, however, can become excessively complicated to determine, and, furthermore, the robustness of the accompanying Newton-Raphson scheme is significantly impaired due to ill-conditioning associated to strain softening [15].

The essence of the Impl-Ex integration method is to combine the advantageous features of both implicit and explicit methods, while, at the same time, minimizing their disadvantages. According to Oliver et al. [15], such a balanced blend is achieved by approximating the derivative in Equation (73) by the derivative appearing in expression Equation (74); i.e. truncating the term $\mathcal{O}\left(\Delta^{2} t_{n}\right)$ in Equation (74), solving then for the derivative term in this equation, and finally substituting the resulting expression in Equation (73), we obtain

$$
\tilde{\xi}_{n+1}=\xi_{n}+\left(\xi_{n}-\xi_{n-1}\right) \frac{\Delta t_{n+1}}{\Delta t_{n}},
$$

where the tilde symbol in $\tilde{\xi}_{n+1}$ indicates that it is the value of the internal variables stemming from the Impl-Ex integration. Equation (75) represents, thus, the Impl-Ex update of the internal variables at $t_{n+1}\left(\tilde{\xi}_{n+1}\right)$ in terms of implicit values computed at $t_{n}$ and $t_{n-1}\left(\xi_{n}\right.$ and $\xi_{n-1}$, respectively). As in explicit methods, the yield condition is not enforced at time $t_{n+1}$. However, in contrast to explicit methods, the accumulated error cannot grow unboundedly, since in each step the implicitly calculated variables contributes to keep the stress close to the yield surfaces. The main restriction on the size of time steps stems from accuracy requirements, as in fully implicit schemes.

The set of state variables defining the size and shape of the yield surfaces at time $t_{n+1}$ can be simply obtained from the corresponding hardening/softening laws using the vector of internal variables derived in Equation (75) as argument, e.g. $\tilde{s}_{1, n+1}=s_{1 \mathrm{~h}}\left(\tilde{\xi}_{n+1}^{h}\right)$. As regards the plastic multipliers, Oliver et al. [15] propose to compute these variables by simply multiplying the corresponding implicit discrete plastic multiplier at $t_{n}$ by the quotient $\Delta t_{n+1} / \Delta t_{n}$, i.e. $\Delta \tilde{\lambda}_{n+1}=$ $\Delta \lambda_{n}\left(\Delta t_{n+1} / \Delta t_{n}\right)$. Although rigorously valid, in principle, only for plasticity models with a single yield surface, ${ }^{*}$ we adopt also this update procedure; hence:

$$
\Delta \tilde{\lambda}_{n+1}^{\beta}=\Delta \lambda_{n}^{\beta} \frac{\Delta t_{n+1}}{\Delta t_{n}}, \quad \beta=e, d, v .
$$

As it will become apparent in the following, considering $\Delta \tilde{\lambda}_{n+1}^{\beta}$ independent of the current deformation affords great simplification of the expression for the algorithmic tangent moduli.

The concept of extrapolation is not applied for computing the Impl-Ex Kirchhoff stress tensor at $t_{n+1}$. Consequently, its update expression follows easily from Equation (45) by simply replacing $\xi_{n+1}^{h}, \xi_{n+1}^{s}$ and $\Delta \lambda_{n+1}^{\beta}$ by their Impl-Ex counterparts, i.e.:

$$
\tilde{\boldsymbol{\tau}}_{n+1}=\tau_{n+1}^{\mathrm{tr}}+\Delta \tilde{\boldsymbol{c}}_{\boldsymbol{e}, n+1}:\left(\mathbf{e}_{n+1}-\breve{\mathbf{e}}_{p, n}\right)-\sum_{\beta=1}^{3} \Delta \tilde{\lambda}_{n+1}^{\beta} \tilde{\boldsymbol{c}}_{\boldsymbol{e}, n+1}: \tilde{\boldsymbol{m}}_{n+1}^{\beta}
$$

\footnotetext{
$\sharp$ The Impl-Ex integration scheme was conceived [15] for integrating numerically elastic-plastic constitutive models with a single yield surface and a single scalar internal variable; in those cases, by properly defining the flow rule, the rate of change of the internal variable can be identified with the continuum plastic multiplier, i.e. $\dot{\xi}=\dot{\lambda}$, and therefore, from (75) one can legitimately write $\Delta \tilde{\lambda}_{n+1}=\Delta \lambda_{n} / \Delta t_{n}$.
} 
In general, obtaining $\tilde{\tau}_{n+1}$ from Equation (77) requires solving a non-linear tensor equation. However, in our case, inspection of Equations (21)-(23) reveals that the plastic flow vector on any of the three yield surfaces bears an affine relation with the Kirchhoff stress tensor

$$
\tilde{\boldsymbol{m}}_{n+1}^{\beta}=\tilde{\boldsymbol{A}}_{n+1}^{\beta}: \tilde{\tau}_{n+1}+\tilde{\boldsymbol{B}}_{n+1}^{\beta},
$$

where the fourth-order and second-order tensors $\tilde{\boldsymbol{A}}_{n+1}^{\beta}$ and $\tilde{\boldsymbol{B}}_{n+1}^{\beta}$, respectively, are defined by

$$
\tilde{\boldsymbol{A}}_{n+1}^{\beta}=2 \boldsymbol{I}_{\mathrm{dev}}+\frac{1}{3} \tilde{u}_{n+1}^{\beta} \mathbf{1} \otimes \mathbf{1}, \quad \tilde{\boldsymbol{B}}_{n+1}^{\beta}=\tilde{v}_{n+1}^{\beta} \mathbf{1} .
$$

The yield surface-dependent parameters $\tilde{u}_{n+1}^{\beta}$ and $\tilde{v}_{n+1}^{\beta}, \beta=e, d, v$, are given, in turn, by the following relationships:

$$
\begin{aligned}
& \tilde{u}_{n+1}^{e}=\frac{2}{3}\left(\tilde{s}_{2, n+1}\right)^{2}, \quad \tilde{u}_{n+1}^{d}=\tilde{u}_{n+1}^{v}=0, \\
& \tilde{v}_{n+1}^{e}=\tilde{v}_{n+1}^{v}=0, \quad \tilde{v}_{n+1}^{d}=\frac{1}{3} \gamma \tilde{c}_{n+1} \alpha .
\end{aligned}
$$

Substituting Equation (78) into Equation (77) and solving for $\tilde{\tau}_{n+1}$ yields

$$
\tilde{\boldsymbol{\tau}}_{n+1}=\tilde{\boldsymbol{R}}_{n+1}^{-1}:\left(\tau_{n+1}^{\mathrm{tr}}+\Delta \tilde{\boldsymbol{c}}_{\boldsymbol{e}, n+1}:\left(\mathbf{e}_{n+1}-\breve{\mathbf{e}}_{p, n}\right)-\tilde{\boldsymbol{c}}_{\boldsymbol{e}, n+1}: \sum_{\beta=1}^{3} \Delta \tilde{\lambda}_{n+1}^{\beta} \tilde{\boldsymbol{B}}_{n+1}^{\beta}\right),
$$

where

$$
\tilde{\boldsymbol{R}}_{n+1}=\boldsymbol{I}+\tilde{\boldsymbol{c}}_{\boldsymbol{e}, n+1}: \sum_{\beta=1}^{3} \Delta \tilde{\lambda}_{n+1}^{\beta} \tilde{\boldsymbol{A}}_{n+1}^{\beta} .
$$

Equation (82) constitutes a closed-form formula for the Kirchhoff stress update at $t_{n+1}$. For implementational purposes, it proves useful to decompose Equation (82) into deviatoric and hydrostatic components

$$
\tilde{\tau}_{n+1}=\operatorname{dev}\left(\tilde{\tau}_{n+1}\right)+\tilde{p}_{n+1} \mathbf{1},
$$

where

$$
\begin{aligned}
\operatorname{dev}\left(\tilde{\tau}_{n+1}\right) & =\frac{\operatorname{dev}\left(\tau_{n+1}^{\mathrm{tr}}\right)+2 \Delta \tilde{\mu}_{n+1}^{e} \operatorname{dev}\left(\mathbf{e}_{n+1}-\breve{\mathbf{e}}_{p, n}\right)}{1+4 \tilde{\mu}_{n+1}^{e} \sum_{\beta=1}^{3} \Delta \tilde{\lambda}_{n+1}^{\beta}}, \\
\tilde{p}_{n+1} & =\frac{p_{n+1}^{\mathrm{tr}}+\Delta \tilde{\kappa}_{n+1}^{e} \operatorname{tr}\left(\mathbf{e}_{n+1}-\breve{\mathbf{e}}_{p, n}\right)+3 \tilde{\kappa}_{n+1}^{e} \sum_{\beta=1}^{3} \Delta \tilde{\lambda}_{n+1}^{\beta} \tilde{v}_{n+1}^{\beta}}{1-\tilde{\kappa}_{n+1}^{e} \sum_{\beta=1}^{3} \Delta \tilde{\lambda}_{n+1}^{\beta} \tilde{u}_{n+1}^{\beta}} .
\end{aligned}
$$

\subsection{Algorithmic elastoplastic tangent moduli}

Attention is focused now on obtaining a closed-form expression for the algorithmic tangent moduli consistent with the Impl-Ex integration scheme, that is, the fourth-order $\tilde{\boldsymbol{c}}_{\boldsymbol{e} \boldsymbol{p}, n+1}$ tensor satisfying the following relationship:

$$
\mathscr{L}_{v} \tilde{\tau}_{n+1}=\tilde{\boldsymbol{c}}_{\boldsymbol{e p}, n+1}: \mathscr{L}_{v} \mathbf{e}_{n+1}=\tilde{\boldsymbol{c}}_{\boldsymbol{e} \boldsymbol{p}, n+1}: \mathbf{d}_{n+1},
$$

wherein the Lie derivative $\mathscr{L}_{v}(\bullet)$ is used to preserve objectivity. The simplicity, in comparison to a standard implicit integration scheme, afforded by the use of the Impl-Ex stress-update scheme arises from the fact that the derivative of the plastic multipliers and the internal variables vanishes, in virtue of the extrapolated character of these variables, that is, $\Delta \tilde{\lambda}_{n+1}^{\beta}$ and $\tilde{\xi}_{n+1}$ do not depend 
on the deformation state at $t_{n+1}$. However, even with this simplification, it takes tedious algebra to derive a closed-form expression for $\tilde{\boldsymbol{c}}_{\boldsymbol{e} \boldsymbol{p}, n+1}$. This derivation is hence relegated to Appendix B, and we simply record here the final expression, which takes the following form:

$$
\tilde{\boldsymbol{c}}_{\boldsymbol{e} \boldsymbol{p}, n+1}=\tilde{\boldsymbol{R}}_{n+1}^{-1}:\left(\tilde{\mathbf{a}}_{\mathbf{e}, n+1}\left(\tilde{\mathbf{e}}_{e, n+1}\right)+\tilde{\boldsymbol{c}}_{\boldsymbol{e}, n+1}:\left(\boldsymbol{I}-\sum_{\beta=1}^{3} \Delta \tilde{\lambda}_{n+1}^{\beta} \tilde{\boldsymbol{z}}_{n+1}^{\beta}\left(\tilde{\tau}_{n+1}\right)\right)\right) .
$$

The isotropic fourth-order tensor $\tilde{\boldsymbol{R}}_{n+1}$ was introduced when deriving the closed-form expression for the stress-update (see Equation (83)). The fourth-order non-symmetric tensor $\tilde{\mathbf{a}}_{\mathbf{e}, n+1}\left(\tilde{\mathbf{e}}_{e, n+1}\right)$ arises, as explained in Appendix B, formulae (B5) and (B10), from applying the Lie derivative to the metric tensor appearing in the definitions of both the elasticity tensor and the plastic flow vectors; these tensors are, thus, large strains contributions. Accordingly, if the corresponding terms are discarded from Equation (88), one obtains the algorithmic tangent operator for the Impl-Ex integration scheme corresponding to a small strain formulation:

$$
\left.\tilde{\boldsymbol{c}}_{\boldsymbol{e p}, n+1}\right|_{\|\mathbf{e}\| \ll 1}=\tilde{\boldsymbol{R}}_{n+1}^{-1}: \tilde{\boldsymbol{c}}_{\boldsymbol{e}, n+1} .
$$

Expanding this equation using Equation (83) gives

$$
\left.\tilde{\boldsymbol{c}}_{\boldsymbol{e p}, n+1}\right|_{\|\mathbf{e}\| \ll 1}=\tilde{\kappa}_{n+1}^{i m x} \mathbf{1} \otimes \mathbf{1}+2 \tilde{\mu}_{n+1}^{i m x} \boldsymbol{I}_{\mathrm{dev}},
$$

where

$$
\begin{aligned}
\tilde{\kappa}_{n+1}^{i m x} & =\frac{\tilde{\kappa}_{n+1}^{e}}{1+3 \tilde{\kappa}_{n+1}^{e} \sum_{\beta=1}^{3} \Delta \tilde{\lambda}_{n+1}^{\beta} \tilde{u}_{n+1}^{\beta}} \\
& =\frac{\tilde{\kappa}_{n+1}^{e}}{1+2 \tilde{\kappa}_{n+1}^{e} \Delta \tilde{\lambda}_{n+1}^{e}\left(\tilde{s}_{2, n+1}\right)^{2}}, \\
\tilde{\mu}_{n+1}^{i m x} & =\frac{\tilde{\mu}_{n+1}^{e}}{1+4 \tilde{\mu}_{n+1}^{e} \sum_{\beta=1}^{3} \Delta \tilde{\lambda}_{n+1}^{\beta}} .
\end{aligned}
$$

Inspection of Equation (90) indicates that, in the small strain setting, the algorithmic tangent operator emanating from the Impl-Ex integration scheme is an isotropic tensor of rank 4, characterized by two parameters, which, in analogy to the notation for the elastic bulk and shear modulus, are denoted by $\tilde{\kappa}_{n+1}^{i m x}$ and $\tilde{\mu}_{n+1}^{i m x}$. Furthermore, since $\Delta \tilde{\lambda}_{n+1}^{\beta} \geqslant 0$, it follows from Equations (91) and (92) that

$$
\begin{aligned}
& \tilde{\kappa}_{n+1}^{i m x} \leqslant \tilde{\kappa}_{n+1}^{e}, \\
& \tilde{\mu}_{n+1}^{i m x} \leqslant \tilde{\mu}_{n+1}^{e},
\end{aligned}
$$

where the inequality or equality holds accordingly as the material is deforming plastically or elastically, respectively.

As alluded to in the introduction, the development of the implicit-explicit integration scheme was prompted by the need to enhance the spectral properties of the algorithmic elastoplastic moduli, whose positive definiteness cannot be guaranteed when using a standard implicit integration scheme, mainly due to the presence of strain softening. Since, according to Equations (91) and (92), $\tilde{\kappa}_{n+1}^{i m x}>0$ and $\tilde{\mu}_{n+1}^{i m x}>0$, it follows then that the algorithmic elastoplastic moduli shown in (90) is positive definite. Another remarkable property of this small strain algorithmic operator is that it does not depend on the deformation state at $t_{n+1}$, hence it can be regarded as step-constant. This means that, if one disregards other sources of non-linearities rather than the material one, the structural tangent stiffness matrix would be constant and, consequently, the convergence to equilibrium states could be achieved in only one iteration per time step. 
One can conclude from the foregoing remark that the positive definiteness of the algorithmic elastoplastic moduli is guaranteed in those situations in which a small strain kinematics is sufficient to yield a realistic description of the deformation state. The ejection stage, which is part of the compaction cycle in which the compacted powder is more liable to develop cracks, falls within these types of situations, since the magnitude of plastic and elastic strains are relatively small, and, besides, the motion of the compact through the die cavity during ejection involves no solid rigid rotations. Therefore, we can assert that, in contrast to the situation that one would encounter if a standard implicit integration scheme is employed, the numerical performance of the algorithm is not impaired by the presence of strain softening, at least in the computation of the response corresponding to the ejection stage.

\section{NUMERICAL RESULTS}

In this section, we examine the performance of the proposed integration algorithm ${ }^{\S \S}$ in two distinctly different situations, namely: (a) the pressing of a cylindrical part, in which hardening behavior dominates the response $\left(\dot{\xi}^{h}>0, \dot{\xi}^{s}=0\right)$; and (b) a fracture test, in which strain localization induced by strain softening $\left(\dot{\xi}^{s}>0\right)$ takes place, while no perceptible density changes occur $\left(\dot{\xi}^{h}=0\right)$. In the first case, results for several time discretizations will be critically compared with the pertinent analytical solution. No analytical data is available for the fracture test; the study in this case will be confined to assess the ability of the algorithm to compute the post-peak response.

Before launching into details, it is convenient to provide an abridged overview of some aspects of the numerical implementation which, although not addressed in this work, are crucial for acquiring a proper grasp of the ensuing computed results. As regards finite element approximation, an updated lagrangian viewpoint has been adopted for describing the motion of the mesh, with a mesh update procedure based on the so-called Particle Finite Element Method (PFEM) (see [27]). This method imposes a limitation concerning element technology: finite elements are to be three-node triangular elements (linear). To avoid the acclaimed inaccuracies that may emerge in the response in using such simple finite elements (e.g. locking), a finite element approximation based on a mixed variational formulation, with displacements and pressure as basic variables, and continuous linear interpolation for both fields, has been implemented. III The localization bandwidth at each quadrature point (see Section 2.3) is calculated as $l_{f}=\sqrt{2 A_{e}}$, where $A_{e}$ is the area of the corresponding triangular element.

\subsection{Pressing of a cylindrical part}

A cylindrical part made of an iron-based Distaloy AE powder, with equal apparent and initial relative densities $\eta_{\mathrm{app}}=\eta_{0}=0.414$, is pressed until reaching a final relative density $\rho=0.98$. The radius of the cylinder is $25 \mathrm{~mm}$, and its initial height $h_{0}=15 \mathrm{~mm}$; see Figure 4 . A downward displacement $\Delta U=-8.72 \mathrm{~mm}$ is prescribed on the top surface of the cylinder; the bottom surface remains stationary and the displacement of the nodes of the lateral surface is restrained in the radial direction (equivalent to a perfectly rigid die). The total time is $\Delta T=1 \mathrm{~s}$; this gives an axial stretch ratio:

$$
\Lambda_{z}(t)=\frac{h(t)}{h_{0}}=1-0.57 t \quad \text { with } t \in[0,1] .
$$

Material parameters can be obtained from the empirical adjustment presented in Section 2.

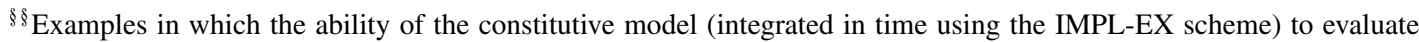
the risk of cracking in practical situations is evaluated can be found in Reference [1].

"II For details on the implementation procedure for the employed mixed formulation, the reader is referred to [28, 29]. 


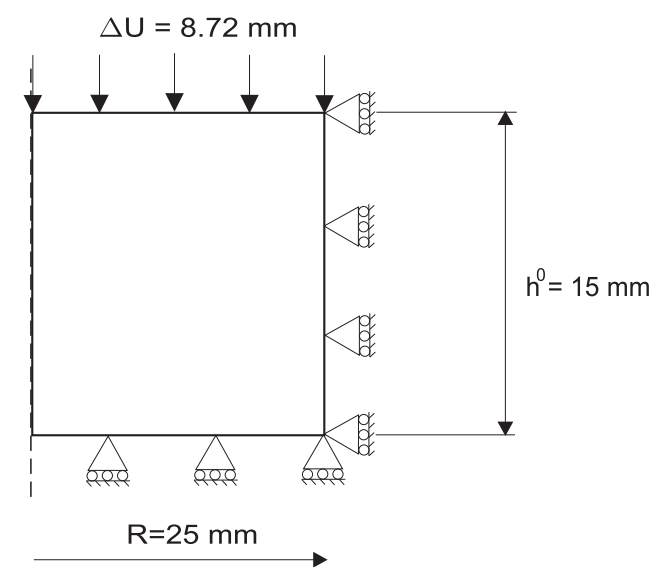

Figure 4. Initial dimensions of the cylindrical part.

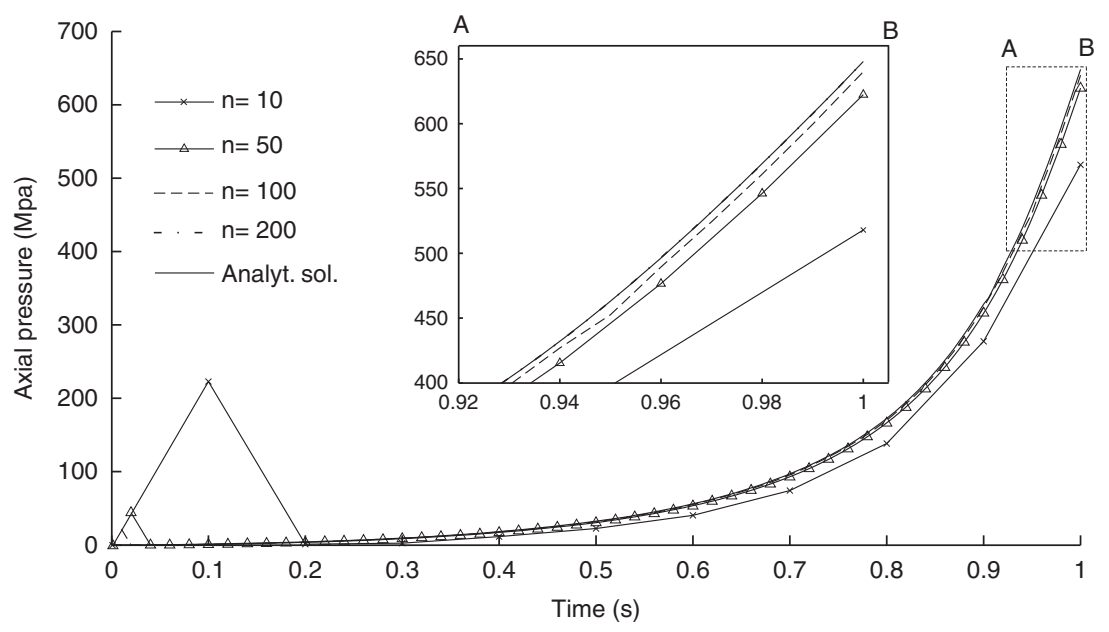

Figure 5. Average axial pressure during pressing versus time. Analytical solution and results for several time steps (uniformly spaced). The final portion of the curves is shown in magnified form.

5.1.1. Study of convergence with refinement of time discretization. Figure 5 shows axial pressure versus time graphs computed using different number of (constant) time steps; the curve corresponding to the analytical solution, derived in Reference [30], and that reads as:

$$
\left|\sigma_{z}(t)\right|=\frac{\left|\tau_{z}\right|}{J}=\frac{\eta_{0} s_{1}(\eta)}{\eta\left(\Lambda_{z}\right)} \sqrt{\frac{2}{3} s_{2}^{2}(\eta)+1}
$$

with $\eta \approx \xi^{h}=\eta_{0} / \Lambda_{z}^{2}$, is also displayed. The sequence of graphs is clearly convergent; the analytical curve and the result with $N=200$ steps are virtually indistinguishable. Inspection of the initial portion of the curves reveals an anomalous behavior for low number of steps. At the very first increment, the solution calculated for the four cases presented an 'overshoot'. The magnitude of the deviation decreases as the time step is reduced, being practically imperceptible for $N=200$ steps. It transpires that this overshooting is not connected with any type of instability, since the computed response returns to the presumably correct course at the second step. For instance, for $N=50$ steps, the axial stress computed at the first increment is largely overestimated (40 MPa, in contrast to the $0.1 \mathrm{MPa}$ predicted with $N=200$ steps). Despite this initial substantial error, the drift from the correct curve in subsequent increments remains bounded, being the magnitude of the maximum pressure (compaction pressure) only $3.5 \%$ below the pressure computed with $N=200$ steps. 


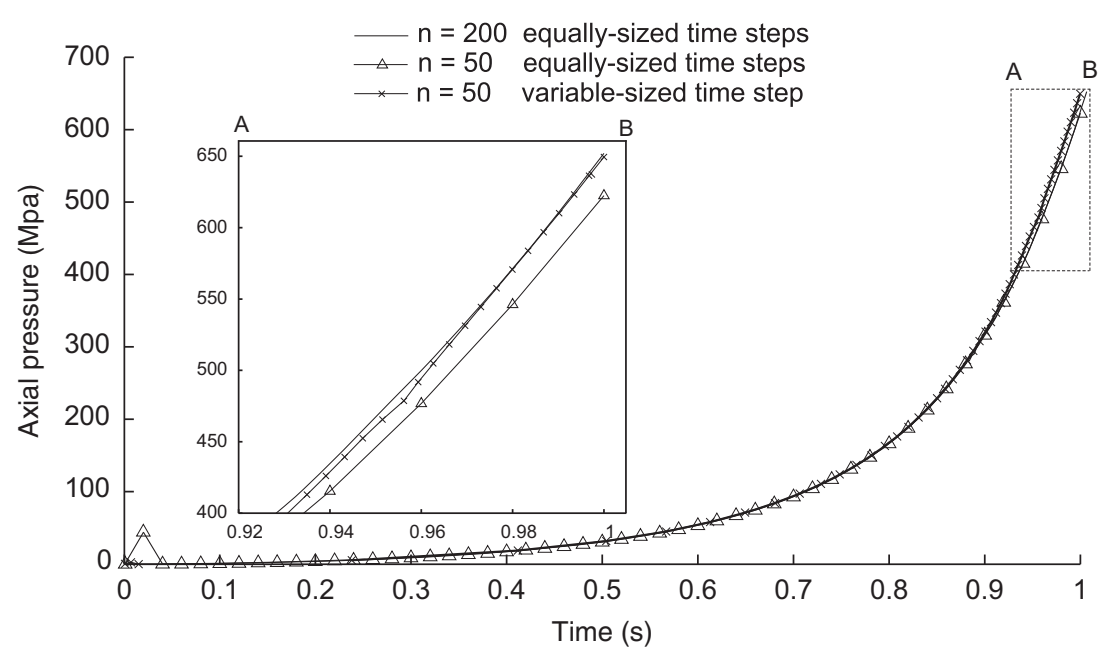

Figure 6. Average axial pressure during pressing versus time. Results for several time steps (constant and variable time steps). The final portion of the curves is shown in magnified form.

The origin of this non-physical overshooting behavior is to be sought in the numerical integration of the constitutive equation, more precisely, in the intricacies of the IMPL-EX integration scheme. As explained in Section 4, the essence of this integration procedure is to obtain the stresses and other state variables at a given time step in terms of variables computed at the previous time step by accomplishing an implicit integration. At the very first increment, obviously, no information is available to carry out this extrapolation. The integration algorithm resolves this inconsistency by further assuming that the plastic multipliers are initially zero. Such an assumption amounts to presume that the body behaves elastically at the first step. This explains why, for the four cases, axial stresses at the first increment lie along a straight line (see Figure 5).

It follows then that the natural way to counteract this overshooting problem is to reduce the time step size. However, diminishing uniformly the step size over the whole time domain is not an efficient practice. It is preferably a 'smart' distribution of the time step length that permits the reduction of the integration error at reduced computational cost. Adequate time step distributions can be obtained by using either adaptive time stepping schemes, of the type described in Reference [15], or from elaborated heuristics taking into account the inherent non-linearity of the material. Here, we employ the one proposed in Reference [15]. In Figure 6, the response obtained using 50 and 200 equally sized spaced time intervals is compared with the solution computed with 50 variable-sized time steps. The size distribution for the non-uniform discretization generated by the adaptive algorithm is shown in Figure 7(b). The relatively small size of the two first intervals is related with the need to overcome the overshooting problem. Observe that the size of the subsequent time steps is directly connected with the compressibility of the material: as the slope of the pressure versus density curve becomes more pronounced, the length of the time step is progressively reduced, so that the incremental changes in stress remains approximately constant. Inspection of Figure 6 indicates that a similar level of accuracy is obtained by using either 200 steps uniformly spaced or 50 steps with sizes non-uniformly distributed. The use of this 'smart' step size distribution has reduced thus the computational effort by a factor of $200 / 50=4$. Finally, Figure 7(b) displays the number of global equilibrium iterations required to achieve convergence at each increment for the case $N=50$ variable time steps. The number of iterations ranges between 1 and 3, resulting in a total number of computational cycles (number of increments times number of iterations) of 66 .

\subsection{Diametral compression test}

The fracture test chosen for assessing the performance of the IMPL-EX algorithm in situations involving strain softening is the diametral compression or Brazilian test. Force is applied over two 

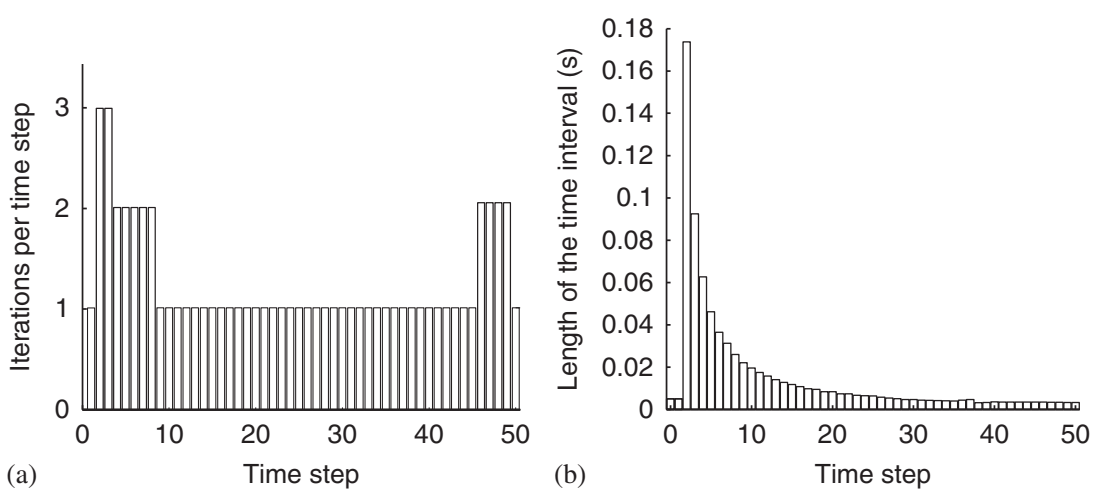

Figure 7. (a) Number of iterations (global scheme) against time step number and (b) length of the time interval versus time step number. Both results correspond to the case $N=50$ (variable) steps shown in Figure 6.

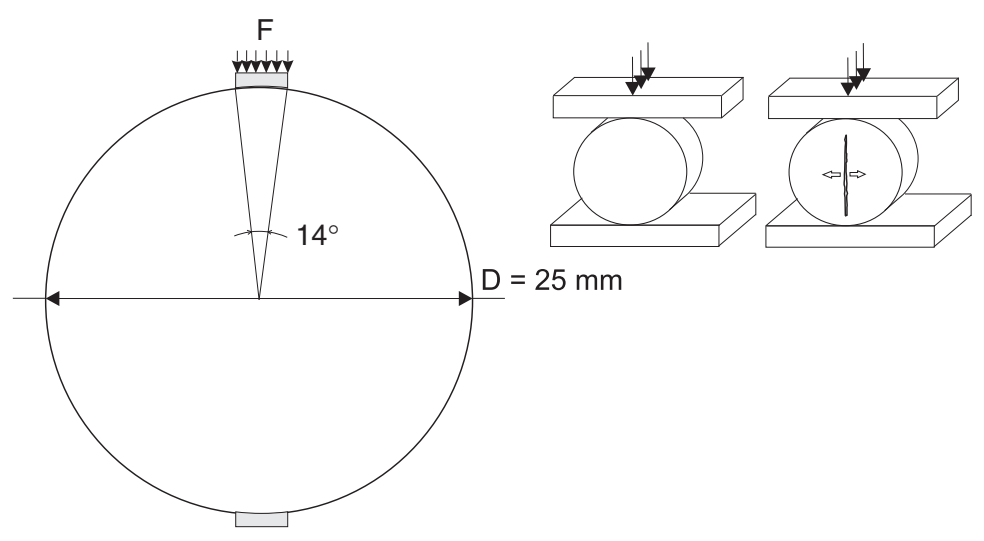

Figure 8. Diametral compression test.

diametrically opposite arcs of angular width $2 \alpha_{B}=14^{\circ}$ (see Figure 8). The fixture tools are modeled as elastic bodies, with Young's modulus $E_{\text {tool }}=2.110^{5} \mathrm{MPa}$ and Poisson's coefficient $\gamma=0.3$. The final dimensions are $D=25 \mathrm{~mm}$ and $t=5 \mathrm{~mm}$, where $D$ denotes the diameter and $t$ the height of the cylindrical specimen. According to Doremus et al. [31], these dimensions $(t / D=0.2)$ ensure that the proviso plane stress can be employed to analyze the stress state during the experiment. For simulating the experiment, a vertical displacement of $0.2 \mathrm{~mm}$ is prescribed upon the top face of the upper fixture tool. The density of the specimen is assumed uniform and equal to $7.21 \mathrm{~g} / \mathrm{cm}^{3}$. The material properties correspond to a Distaloy AE powder with apparent and theoretical density 3.10 and $7.48 \mathrm{~g} / \mathrm{cm}^{3}$, respectively, and can thus be obtained from the curve-fitting equations presented in Section 2 by simply setting the internal hardening parameter to $\xi^{h} \approx \eta=7.21 / 7.48=0.96$.

Convergence analysis under refinement of the discretized time is carried out by examining $F-v$ plots. The vertical deflection $v$ corresponds to the displacement prescribed upon the nodes located at the top face of the fixture tool; the applied load $F$ is obtained as the sum of the forces at these nodes. The mesh used for the computations is displayed in Figure 9; the higher mesh density in the vertical central band is dictated by foresight of the fact that the dominant crack will be located along the loaded diameter.

Figure 10 shows the result of the convergence study. The plot of applied load versus deflection takes essentially the same form in all the analyzed cases, the only substantial difference being the post-peak behavior. The branch OA corresponds to linear elasticity. After point A, a slight decrease in slope occurs. This subtle change of stiffness indicates crack initiation at the center of the disk (see Figure 11). The development of the central dominant crack progresses until the maximum force is reached (point B in case $N=800$ ). The magnitude of the maximum force estimated using 


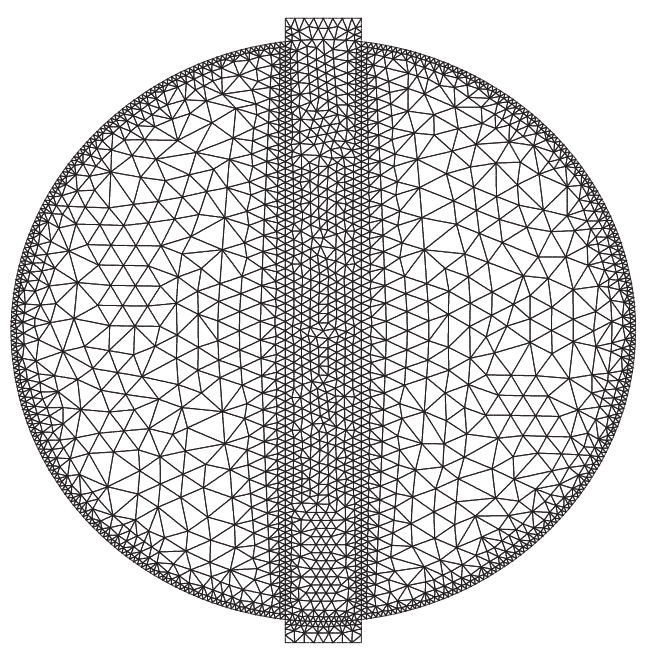

Figure 9. Mesh layout.

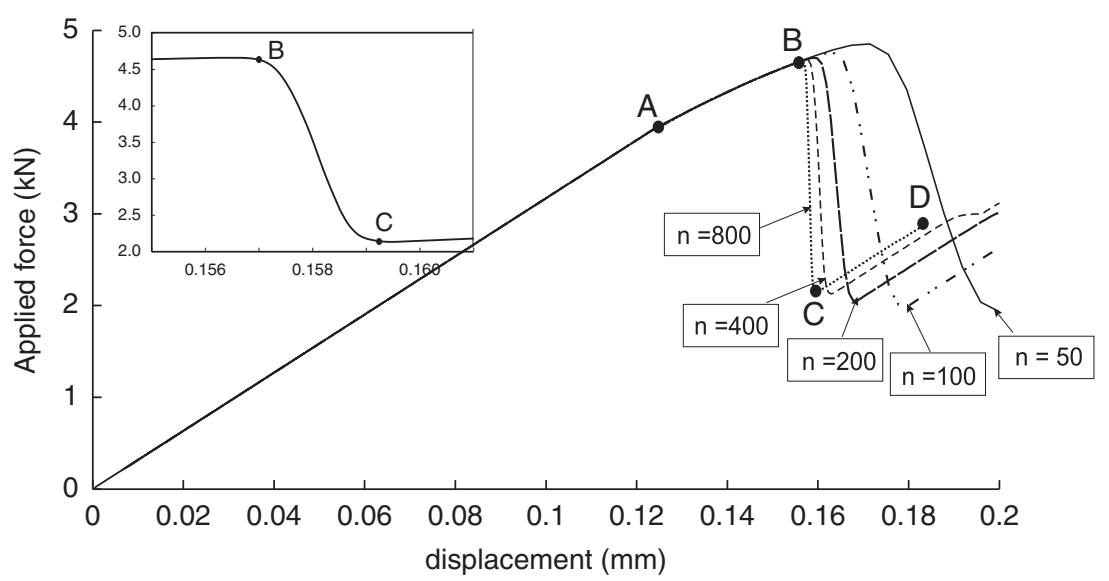

Figure 10. Applied load versus deflection. Results for several time steps.

$N=50$ steps differs from that calculated with $N=800$ by only $4.3 \%$. Following the attainment of the peak-load, the central fiber suddenly ceases to contribute to the rigidity in the vertical direction, and the computed force drops rapidly (point $\mathrm{C}$ ). The abruptness of this decay is accentuated as the time step size is reduced. The enlarged view of path BC, shown in the upper-left part of Figure 10, reveals that the transition from $\mathrm{B}$ to $\mathrm{C}$, although certainly quite abrupt, is not instantaneous; that is, several time steps are required in its computation. ${ }^{\| I}$ The post-peak ascending branch (CD) corresponds, approximately, to the response of a specimen split into two halves.

These numerical results serve as convincing proof that the solution provided by the Impl-Ex integration algorithm converges as the length of the time step is reduced also in situations entailing strain softening. A remarkable feature is that one iteration per step, for the five cases presented, was required to achieve convergence to equilibrium states; this confirms the robustness of the Impl-Ex integration scheme (see Section 4). Not least among its merits is the ability to compute the post-peak response (curve BCD in Figure 10). A standard implicit integration scheme would probably encounter serious difficulties ${ }^{* * *}$ in advancing the solution beyond this critical point, since

"III An instantaneous drop would correspond to a perfectly brittle response. A force versus displacement graph that *** exhibits such feature warns of trouble with potential snap-back behavior [33].

***Especially if the solution algorithm is not enhanced by cumbersome continuation methods (see e.g. [33, 34]). 

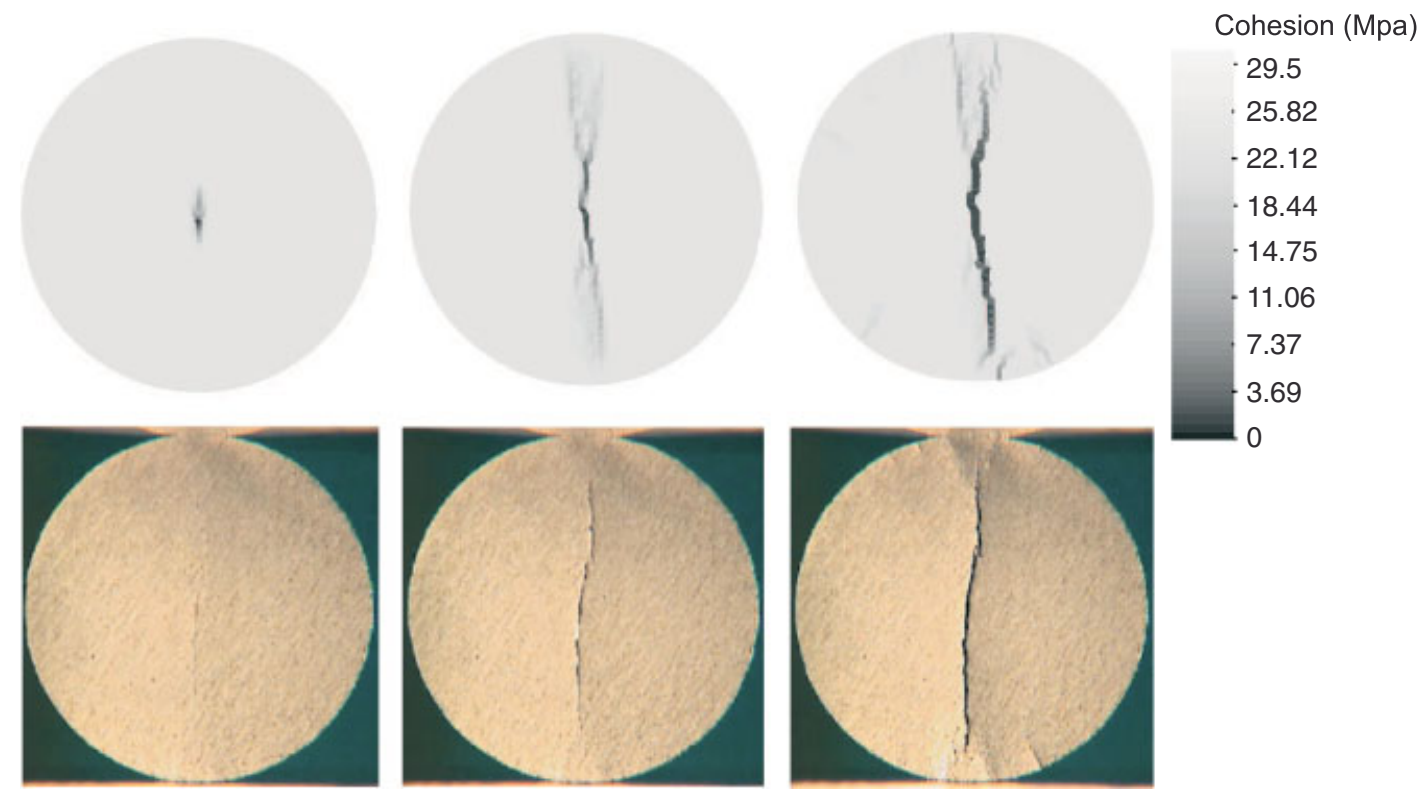

(c)

Figure 11. Contour plots of computed cohesion corresponding to: (a) initiation of the crack; (b) point of maximum load; and (c) end of the loading process. Below, comparison with images recorded experimentally [32].

strain softening invariably impair, to a greater or lesser degree, the conditioning of the implicit tangent stiffness matrix, and hence the computability of the response.

In order to facilitate the comparison, equally spaced intervals have been used in the simulation of this test. However, we should note that considerable gain in computational efficiency could have been achieved by means of the adaptive time-stepping scheme mentioned in the previous section. Since, under elastic conditions, the IMPLE-EX and the fully implicit integration procedures yield identical results, the elastic response OA in Figure 10 could have been obtained in just a single step.

\section{CONCLUSIONS}

Standard implicit and explicit integration schemes have complementary advantages and drawbacks; accordingly, a combined or hybrid (implicit-explicit) approach should furnish superior results. The goal of this paper was to explore this issue for the particular case of the integration of a complex, multisurface constitutive model able to describe both the densification of metal powders and the cracking of green compacts in $\mathrm{P} / \mathrm{M}$ cold compaction processes. The hybrid integration procedure employed for this purpose has been the so-called IMPL-EX scheme, pioneered by Oliver et al. $[15,17]$. First, it has been shown that the use of the IMPL-EX method eliminates the adverse effects of softening-induced non-positive definite algorithmic elastoplastic moduli, which is one the flaws exhibited by fully implicit solutions. Unconditional stability, on the other hand, is inherited from the implicit part of the algorithm; the only restriction in the size of time step stems, thus, from accuracy considerations, as in fully implicit methods. Consequently, if used in conjunction with an appropriate adaptive time stepping scheme, the IMPL-EX integration scheme can offer an efficient solution to the trade-off between robustness and computational time requirements.

The distinguishing feature of the IMPL-EX integration strategy is that it does entail the solution, at each time increment, of the non-linear system of equations stemming from the implicit backward Euler difference scheme. A major contribution of this paper has been the development of an apparently novel, fractional step method-based (FSM) algorithm for solving these equations. It has been demonstrated in a mathematically sound manner that the proposed algorithm provides a remarkable 
aspect of robustness, inasmuch as it converges to the solution regardless of the values of the material properties and the location of the elastic trial stress in relation with respect to the prevailing yield surfaces; furthermore, its convergence is even faster when the trial stress is placed far from the yield surface. The algorithm dispenses with the need for computing gradients of any involved function (yield surfaces, hardening/softening laws) and, hence, it seems suitable for solving the return-mapping equations of complex constitutive models with nonsmooth hardening/softening laws and yield surfaces. Attempts to generalize and apply the FSM algorithm to other material models, however, should be tempered against the computational cost of undertaking the predictor step (projection of the trial stress state back to a fixed yield surface): should this step require an iterative process on its own, the application of the FSM algorithm might prove computationally inefficient, since local convergence would involve two (nested) iterations. Finally, a subtle, yet significant, advantage of using the proposed FSM algorithm is the considerable amount of flexibility it affords in the corresponding computational implementation. Indeed, yield of plastic potential surfaces, on the one hand, and evolutionary equations and hardening/softening laws, on the other, are treated separately. This means that the implementation of, e.g. a distinct hardening/softening law can be conducted in a modular fashion: the predictor step equations would remain unaltered, and only pertinent changes on the equations for the corrector step would be needed.

\section{APPENDIX A: CONVERGENCE ANALYSIS FOR THE FSM PREDICTOR-CORRECTOR SCHEME}

This appendix is devoted to study the properties of the sequence $\left\{q^{(k)}\right\}_{k=0}^{\infty}$ defined by the following recursion formula (see Section 3.1):

$$
\frac{\overbrace{}^{(k+1)}\left(q^{(k+1)}+\alpha p_{n+1}^{\mathrm{tr}}\right)}{\frac{\Theta_{p d}\left(q^{(k+1)}\right)}{2} \chi\left(q_{n+1}^{\mathrm{tr}}-q^{(k+1)}\right)+q^{(k+1)}}=\frac{\Theta_{c r}\left(q^{(k)}\right)}{c_{n}}
$$

with $q^{(0)}=q_{n+1}^{\mathrm{tr}}$, in the interval $\left[q_{\min }, q_{n+1}^{\mathrm{tr}}\right]$, where $q_{\min }=\max \left(0,-\alpha p_{n+1}^{\mathrm{tr}}\right)$.

\section{A.1 Sufficient conditions for convergence}

Proposition A1

Let $\left\{q^{(k)}\right\}$ be the sequence defined by the recursion formula

$$
\Theta_{p d}\left(q^{(k+1)}\right)=\Theta_{c r}\left(q^{(k)}\right), \quad q^{(0)}=q_{n+1}^{\mathrm{tr}} .
$$

Assume that:

(I) $\Theta_{p d}(q)$ and $\Theta_{c r}(q)$ are continuously differentiable in $\left[q_{\min }, q_{n+1}^{\mathrm{tr}}\right]$.

(II) $\Theta_{p d}(q)$ and $\Theta_{c r}(q)$ are monotonically increasing functions, i.e. $\dot{\Theta}_{p d}(q) \geqslant 0$ and $\Theta_{c r}(q) \geqslant 0$, $\forall q \in\left[q_{\min }, q_{n+1}^{\mathrm{tr}}\right]$.

(III) $\Theta_{p d}\left(q_{n+1}^{\mathrm{tr}}\right) \geqslant \Theta_{c r}\left(q_{n+1}^{\mathrm{tr}}\right)$ and $\Theta_{p d}\left(q_{\min }\right) \leqslant \Theta_{c r}\left(q_{\min }\right)$.

(IV) There exists an unique solution $q^{*}$ in the interval $\left[q_{\min }, q_{n+1}^{\mathrm{tr}}\right]$ to the equation $\Theta_{p d}=\Theta_{c r}$.

Then, the sequence $\left\{q^{(k)}\right\}$ converges to $q^{*}$.

Proof

The proof is carried out by showing that $\left\{q^{(k)}\right\}$ is a bounded monotonic (decreasing) sequence. In virtue of assumption $I$, we can expand $\Theta_{p d}\left(q^{(k+1)}\right)$ and $\Theta_{p d}\left(q^{(k+1)}\right)$ in the Taylor series 
about $q^{(k)}$ :

$$
\begin{aligned}
& \Theta_{p d}\left(q^{(k+1)}\right)=\Theta_{p d}\left(q^{(k)}\right)+\dot{\Theta}_{p d}\left(\eta_{p}\right)\left(q^{(k+1)}-q^{(k)}\right), \quad \eta_{p} \in\left[q^{(k+1)}, q^{(k)}\right], \\
& \Theta_{c r}\left(q^{(k+1)}\right)=\Theta_{c r}\left(q^{(k)}\right)+\dot{\Theta}_{c r}\left(\eta_{c}\right)\left(q^{(k+1)}-q^{(k)}\right), \quad \eta_{c} \in\left[q^{(k+1)}, q^{(k)}\right] .
\end{aligned}
$$

Employing the recursion formula, we may write the above equations as

$$
\begin{aligned}
\Theta_{c r}\left(q^{(k)}\right)-\Theta_{p d}\left(q^{(k)}\right) & =\dot{\Theta}_{p d}\left(\eta_{p}\right)\left(q^{(k+1)}-q^{(k)}\right), \quad \eta_{p} \in\left[q^{(k+1)}, q^{(k)}\right], \\
\Theta_{c r}\left(q^{(k+1)}\right)-\Theta_{p d}\left(q^{(k+1)}\right) & =\dot{\Theta}_{c r}\left(\eta_{c}\right)\left(q^{(k+1)}-q^{(k)}\right), \quad \eta_{c} \in\left[q^{(k+1)}, q^{(k)}\right] .
\end{aligned}
$$

The monotonic character of $\left\{q^{(k)}\right\}$, i.e. $q^{(k+1)} \leqslant q^{(k)}$ for all $k=0,1,2 \ldots$ is proved by induction on $k$. The result holds trivially for $k=0, q^{(1)} \leqslant q^{(0)}$, as can be deduced from assumptions II and III and by evaluating (A4):

$$
\begin{gathered}
\Theta_{c r}\left(q^{(0)}\right)-\Theta_{p d}\left(q^{(0)}\right)=\dot{\Theta}_{p d}\left(\eta_{p}\right)\left(q^{(1)}-q^{(0)}\right) \Rightarrow \\
\Theta_{c r}\left(q_{n+1}^{\mathrm{tr}}\right)-\Theta_{p d}\left(q_{n+1}^{\mathrm{tr}}\right) \leqslant 0, \quad \dot{\Theta}_{p d}\left(\eta_{p}\right) \geqslant 0 \Rightarrow q^{(1)} \leqslant q^{(0)} .
\end{gathered}
$$

Therefore, assume now that the result is also true for some $k=i$, that is, $q^{(i)} \leqslant q^{(i-1)}$. Combining (A4) and (A5), we obtain

$$
q^{(i+1)}-q^{(i)}=\frac{\dot{\Theta}_{c r}\left(\eta_{c}\right)}{\dot{\Theta}_{p d}\left(\eta_{p}\right)}\left(q^{(i)}-q^{(i-1)}\right) .
$$

Since both $\dot{\Theta}_{c r}$ and $\dot{\Theta}_{p d}$ are nonnegative for all $q \in\left[q_{\min }, q_{n+1}^{\mathrm{tr}}\right]$ (assumption II), it follows immediately that $q^{(i+1)}-q^{(i)} \leqslant 0$.

To complete the proof, we shall show that $q^{(k)} \in\left[q_{\min }, q_{n+1}^{\mathrm{tr}}\right]$ for all $k$, i.e. the sequence is bounded, and hence convergent. To this end, we shall argue by contradiction. Suppose that there exists $k>0$ such that $q^{(k)} \in\left[q_{\min }, q^{*}\right]$. From (A4), the following property can be deduced:

$$
\Theta_{c r}\left(q^{(k)}\right) \leqslant \Theta_{p d}\left(q^{(k)}\right)
$$

for all $k$. Since from assumption III $\Theta_{p d}\left(q_{\min }\right)-\Theta_{c r}\left(q_{\min }\right) \leqslant 0$, we know by the intermediate value problem that $\Theta_{p d}-\Theta_{c r}$ has a zero in $\left[q_{\min }, q^{(k)}\right]$, a fact that contradicts assumption IV, hence $q^{(k)} \in\left[q_{\min }, q_{n+1}^{\mathrm{tr}}\right]$ for all $k$. Furthermore, since the sequence is decreasing, it follows immediately that $q^{*}$ is its limit, as asserted.

We are now confronted with the task of ascertaining whether the particular functional forms of $\Theta_{c r}$ and $\Theta_{p d}$ (see Equations (65) and (69), respectively) satisfy the sufficient conditions listed in the foregoing result. As regards the condition of continuity and differentiability, notice that both $\Theta_{p d}$ and $\Theta_{c r}$ are rational functions of $q$ :

$$
\Theta_{p d}=\frac{N_{p d}(q)}{D_{p d}(q)}, \quad \Theta_{c r}=\frac{N_{c r}}{D_{c r}(q)} .
$$

Since $D_{c r}(q) \neq 0$ for all $q \in\left[q_{\min }, q_{n+1}^{\mathrm{tr}}\right], \Theta_{c r}(q)$ is continuously differentiable on the interval $\left[q_{\min }, q_{n+1}^{\mathrm{tr}}\right]$. For $q_{n+1}^{\mathrm{tr}}=0$, the predictor function $\Theta_{p d}$ has a singularity at $q=0$, i.e. $D_{p d}(0)=0$. However, as may be inferred from Equations (63) and (68), in this case the solution to the returnmapping algorithm is trivial, since the internal variable does not evolve and the trial stress is simply 'returned' to the Drucker-Prager apex, i.e.:

$$
q_{n+1}^{\mathrm{tr}}=0 \Longrightarrow q_{n+1}=0, \quad c_{n+1}=c_{n}, \quad p_{n+1}=\frac{c_{n}}{\alpha} .
$$

Thus, in order to ensure also the continuity and differentiability of $\Theta_{p d}$, the particular case $q_{n+1}^{\text {tr }}=0$ is henceforth excluded from the analysis. 
Condition II states that $\dot{\Theta}_{c r}(q) \geqslant 0$ and $\dot{\Theta}_{p d}(q) \geqslant 0$. The verification for $\Theta_{c r}$ is straightforward:

$$
\dot{\Theta}_{c r}(q)=\frac{c_{n}\left|\hat{H}_{0, n}\right|}{c_{\mathrm{h}, n}\left(1+\frac{\left|\hat{H}_{0, n}\right|}{c_{\mathrm{h}, n}}\left(q_{n+1}^{\mathrm{tr}}-q^{(k)}\right)\right)^{2}} \geqslant 0 \quad \forall q .
$$

To study the sign of the derivative of $\Theta_{p d}$, we can write

$$
\Theta_{p d}(q)=\frac{q}{\frac{1}{2} \chi\left(q_{n+1}^{\mathrm{tr}}-q\right)+q}\left(q+\alpha p_{n+1}^{\mathrm{tr}}\right)=F(q)\left(q+\alpha p_{n+1}^{\mathrm{tr}}\right),
$$

where $F(q) \in[0,1]$ for $q \in\left[q_{\min }, q_{n+1}^{\mathrm{tr}}\right]$. Using the product rule, we obtain

$$
\dot{\Theta}_{p d}(q)=\dot{F}(\overbrace{q+\alpha p_{n+1}^{\text {tr }}}^{>0})+F .
$$

Since $\chi \geqslant 0$, the derivative of $F$

$$
\dot{F}=\frac{1}{2} \frac{\chi q_{n+1}^{\mathrm{tr}}}{\left(\frac{1}{2} \chi\left(q_{n+1}^{\mathrm{tr}}-q\right)+q\right)^{2}}
$$

is also positive for all $q$. Hence, it follows from Equation (A13) that $\dot{\Theta}_{p d}(q) \geqslant 0$ for all $q \in$ $\left[q_{\min }, q_{n+1}^{\mathrm{tr}}\right]$.

The condition III at the extremes of the interval is also satisfied:

$$
\begin{aligned}
\Theta_{p d}\left(q_{n+1}^{\mathrm{tr}}\right)-\Theta_{c r}\left(q_{n+1}^{\mathrm{tr}}\right) & =q_{n+1}^{\mathrm{tr}}+\alpha p_{n+1}^{\mathrm{tr}}-c_{n}=\phi_{n+1}^{d, \mathrm{tr}}>0, \\
\Theta_{p d}\left(q_{\mathrm{min}}\right)-\Theta_{c r}\left(q_{\mathrm{min}}\right) & =0-\frac{c_{n}}{1+\frac{\left|\hat{H}_{0, n}\right|}{c_{\mathrm{h}, n}}\left(q_{n+1}^{\mathrm{tr}}-q_{\mathrm{min}}\right)} \leqslant 0 .
\end{aligned}
$$

Finally, we prove in the following that the function $\Theta(q)=\Theta_{p d}(q)-\Theta_{c r}(q)$ has an unique zero in the interval $\left[q_{\min }, q_{n+1}^{\text {tr }}\right]$ (condition $I V$ ). In turn, this result provides the assurance that the solution exists when $\rrbracket_{n+1}^{\text {act,tr }}=\{d\}$.

Proposition A2

The function defined as $\Theta(q)=\Theta_{p d}(q)-\Theta_{c r}(q)$, with $\Theta_{p d}$ and $\Theta_{p d}$ given in Equations (69) and (65), respectively, has an unique zero in the interval $\left[q_{\min }, q_{n+1}^{\mathrm{tr}}\right]$.

Proof

Multiplying $\Theta=0$ by the denominators of $\Theta_{p d}$ and $\Theta_{c r}$ (which are non-vanishing $\forall q \in$ $\left.\left[q_{\min }, q_{n+1}^{\text {tr }}\right]\right)$, a cubic polynomial $R(q)=\sum_{i=0}^{3} r_{i} q^{i}=0$ is obtained. After elementary manipulations, the coefficients $\left(r_{i}, i=0 \ldots 3\right)$ are found to be given by:

$$
\begin{aligned}
& r_{3}=-\frac{\left|\hat{H}_{0, n}\right|}{c_{\mathrm{h}, n}}, \quad r_{2}=\left(1+\frac{\left|\hat{H}_{0, n}\right|}{c_{\mathrm{h}, n}}\left(q_{n+1}^{\mathrm{tr}}-\alpha p_{n+1}^{\mathrm{tr}}\right)\right), \\
& r_{0}=-\frac{1}{2} c_{n} q_{n+1}^{\mathrm{tr}} \chi, \quad r_{1}=r_{2}-c_{n}\left(1-\frac{1}{2} \chi\right) .
\end{aligned}
$$

Evaluation of the polynomial $R$ at the extremes of the interval yields

$$
\begin{aligned}
& R\left(q_{\mathrm{min}}\right)=-c_{n} \overbrace{\left(\frac{1}{2} \chi\left(q_{n+1}^{\mathrm{tr}}-q_{\min }\right)+q_{\min }\right.}^{>0} \leqslant 0, \\
& R\left(q_{n+1}^{\mathrm{tr}}\right)=q_{n+1}^{\mathrm{tr}}\left(q_{n+1}^{\mathrm{tr}}+\alpha p_{n+1}^{\mathrm{tr}}\right)-c_{n}=q_{n+1}^{\mathrm{tr}} \phi_{n+1}^{d, \mathrm{tr}} \geqslant 0 .
\end{aligned}
$$


Thus, since $R\left(q_{\mathrm{min}}\right)$ and $R\left(q_{n+1}^{\mathrm{tr}}\right)$ have opposite signs, we know by the intermediate value theorem that there exist $n$ roots of $R$ in $\left[q_{\min }, q_{n+1}^{\text {tr }}\right]$, with $n=1$ or $n=3$. The signs of $r_{0}$ and $r_{3}$ indicate that $R(0)<0$ and $R(-\infty)>0$. Therefore, following the same logic as above, $R=0$ have $m$ negative roots, with $m=1$ or $m=3$. If we suppose that $n=3$, then the total number of zeros would be $n+m=4$ or $n+m=6$, which cannot be true, since the polynomial is cubic. Hence, $R$ has only one root in $\left[q_{\min }, q_{n+1}^{\mathrm{tr}}\right]$, and, consequently, $\Theta$ possesses an unique zero in $\left[q_{\min }, q_{n+1}^{\mathrm{tr}}\right]$, as asserted.

Therefore, we conclude that the functional forms of $\Theta_{c r}$ and $\Theta_{p d}$, given in Equations (69) and (65), respectively, fulfill the sufficient conditions for the sequence defined by the recursion formula (70) to converge.

\section{A.2 Rate of convergence}

\section{Proposition A3}

Let $\left\{q^{(k)}\right\}$ be the convergent sequence defined by the recursion formula $\Theta_{p d}\left(q^{(k+1)}\right)=\Theta_{c r}\left(q^{(k)}\right)$. Let $q^{*}$ denote the limit of this sequence and consider an interval $\left[q^{*}, \beta\right] \subset\left[q^{*}, q_{n+1}^{\mathrm{tr}}\right]$ such that

$$
\frac{\dot{\Theta}_{p d}(\eta)}{\dot{\Theta}_{c r}(\eta)}<1
$$

for all $\eta \in\left[q^{*}, \beta\right]$. Let $N$ be a positive integer such that $q^{(N)} \leqslant \beta$. Then, the error, denoted as $e=q-q^{*}$, decreases for all $k \geqslant N$, being the rate of this decrease linear.

Proof

Expressing $\Theta_{p d}\left(q^{(k+1)}\right)$ and $\Theta_{c r}\left(q^{(k)}\right)$ in the Taylor series about $q^{*}$ and truncating after the firstorder term yields:

$$
\Theta_{p d}\left(q^{(k+1)}\right)=\Theta_{p d}\left(q^{*}\right)+\dot{\Theta}_{p d}\left(\eta_{p}\right) \overbrace{q^{(k+1)}-q^{\left(q^{*}\right)}}^{\mathrm{e}^{(k+1)}}, \quad \eta_{p} \in\left[q^{*}, q^{(k+1)}\right]
$$

and

$$
\Theta_{c r}\left(q^{(k)}\right)=\Theta_{c r}\left(q^{*}\right)+\dot{\Theta}_{c r}\left(\eta_{c}\right)(\overbrace{q^{(k+1)}-q^{(k)}}^{\mathrm{e}^{(k)}}), \quad \eta_{c} \in\left[q^{*}, q^{(k)}\right]
$$

respectively. Subtracting Equation (A23) from Equation (A22) gives:

$$
\Theta_{p d}\left(q^{(k+1)}\right)-\Theta_{c r}\left(q^{(k)}\right)=\left(\Theta_{p d}\left(q^{*}\right)-\Theta_{c r}\left(q^{*}\right)\right)+\dot{\Theta}_{p d}\left(\eta_{p}\right) e_{(k+1)}-\dot{\Theta}_{c r}\left(\eta_{c}\right) e_{(k)} .
$$

Since $\Theta_{p d}\left(q^{*}\right)=\Theta_{c r}\left(q^{*}\right)$ and $\Theta_{p d}\left(q^{(k+1)}\right)=\Theta_{c r}\left(q^{(k)}\right)$, after solving for $e_{(k+1)}$ we obtain

$$
e_{(k+1)}=\frac{\dot{\Theta}_{c r}\left(\eta_{c}\right)}{\dot{\Theta}_{p d}\left(\eta_{p}\right)} e_{(k)}
$$

For $k>N, q^{(k)}, q^{(k-1)} \in\left[q^{*}, \beta\right]$. Then, the error decreases by a factor $\dot{\Theta}_{c r}\left(\eta_{p}\right) / \dot{\Theta}_{p d}\left(\eta_{c}\right), \eta_{p} \in$ $\left[q^{*}, q^{(k+1)}\right], \eta_{c} \in\left[q^{*}, q^{(k)}\right]$. Equation (A25) further shows that $e_{(k+1)}$ depends linearly on $e_{(k)}$.

Thus, the above proposition shows that the rate of convergence is linear in a neighborhood of the solution in which $\dot{\Theta}_{c r}>\dot{\Theta}_{p d}$. It is straightforward to prove, by contradiction, that this condition of greater slope of the predictor curve in some $\left[q^{*}, \beta\right], \beta \in\left[0, q_{n+1}^{\text {tr }}-q^{*}\right]$ always holds. Suppose that $\dot{\Theta}_{c r}\left(q^{*}\right)<\dot{\Theta}_{p d}\left(q^{*}\right)$. The continuity assumption implies that, for some $\varepsilon>0, \Theta_{p d}\left(q^{*}+\varepsilon\right)<\Theta_{c r}\left(q^{*}+\right.$ $\varepsilon)$, which is a contradiction, since $\Theta_{p d}(q) \geqslant \Theta_{c r}(q)$ for all $q \in\left[q^{*}, q_{n+1}^{\mathrm{tr}}\right]$. 
The so-called asymptotic convergence factor [25] $\dot{\Theta}_{c r} / \dot{\Theta}_{p d}$ can be estimated grossly using the mean value theorem:

$$
\begin{aligned}
\frac{\Theta_{c r}}{\Theta_{p d}} & =\frac{\Theta_{c r}\left(q_{n+1}^{\mathrm{tr}}\right)-\Theta_{c r}\left(q_{\min }\right)}{\Theta_{p d}\left(q_{n+1}^{\mathrm{tr}}\right)-\Theta_{p d}\left(q_{\min }\right)} \\
& =\frac{\left|\hat{H}_{0, n}\right| / c_{\mathrm{h}, n}\left(q_{n+1}^{\mathrm{tr}}-q_{\min }\right)}{1+\left|\hat{H}_{0, n}\right| / c_{\mathrm{h}, n}\left(q_{n+1}^{\mathrm{tr}}-q_{\min }\right)} \frac{1}{\frac{\phi_{n+1}^{d, \mathrm{tr}}}{c_{n}}+1} .
\end{aligned}
$$

\section{A.3 Quadratically convergent method}

By simply modifying the equation for the update of cohesion in the corrector step, it is plausible to devise, in the light of the predictor-corrector philosophy, a method with a higher rate of convergence. The corresponding sequence is defined by the recursion formulae

$$
\begin{aligned}
\Theta_{p d}\left(q^{(2 k-1)}\right) & =\Theta_{c r}\left(q^{(2 k-2)}\right), \\
\Theta_{c r}\left(q^{(2 k)}\right) & =\Theta_{p d}\left(q^{(2 k-1)}\right)+\dot{\Theta}_{p d}\left(q^{(2 k-1)}\right)\left(q^{(2 k)}-q^{(2 k-1)}\right)
\end{aligned}
$$

with $k=1,2 \ldots$ and $q^{(0)}$. The analysis of the convergence of this sequence is undertaken in a similar manner to that presented in Proposition A3. ${ }^{\dagger \dagger}$ We simply quote the final result (for $k \rightarrow \infty$ ):

$$
\begin{aligned}
e_{(2 k-1)} & =\frac{\dot{\Theta}_{c r}\left(q^{*}\right)}{\dot{\Theta}_{p d}\left(q^{*}\right)} e_{(2 k-2)}, \\
e_{(2 k)} & =\frac{1}{2} \frac{\ddot{\Theta}_{c r}\left(q^{*}\right)}{\dot{\Theta}_{p d}\left(q^{*}\right)-\dot{\Theta}_{c r}\left(q^{*}\right)} e_{(2 k-1)}^{2} .
\end{aligned}
$$

Combining Equations (A29) and (A30), we obtain

$$
e_{(2 k)}=\frac{1}{2} \frac{\ddot{\Theta}_{c r}\left(q^{*}\right)}{\dot{\Theta}_{p d}\left(q^{*}\right)-\dot{\Theta}_{c r}\left(q^{*}\right)}\left(\frac{\dot{\Theta}_{c r}\left(q^{*}\right)}{\dot{\Theta}_{p d}\left(q^{*}\right)}\right)^{2} e_{(2 k-2)}^{2} .
$$

The above equation reveals that the rate of convergence of this modified FSM algorithm is quadratic. However, in return for its enhanced convergence, a necessary condition for the sequence defined by Equations (A29) and (A30) to be monotonic decreasing is that $\ddot{\Theta}_{p d}>0$ in a neighborhood of the solution, i.e. the predictor function must be convex. To check whether this condition is satisfied, (A13) is differentiated with respect to $q$, yielding

$$
\ddot{\Theta}_{p d}=2 \dot{F}+\ddot{F}\left(q+\alpha p_{n+1}^{\mathrm{tr}}\right) .
$$

After some manipulation, $\ddot{F}$ can be expressed as

$$
\ddot{F}=\frac{-2(1-1 / 2 \chi)}{q(1-1 / 2 \chi)+1 / 2 \chi q_{n+1}^{\mathrm{tr}}} \dot{F} .
$$

Substitution of the above into (A32) leads to

$$
\ddot{\Theta}_{p d}=2 \dot{F}\left(1-\frac{-(1-1 / 2 \chi)}{q(1-1 / 2 \chi)+1 / 2 \chi q_{n+1}^{\mathrm{tr}}}\left(q+\alpha p_{n+1}^{\mathrm{tr}}\right)\right) \text {. }
$$

\footnotetext{
$\overline{t^{\dagger}}$ The only difference lies in that, in (A28), $\Theta_{p d}\left(q^{(2 k-1)}\right)$ is expanded in a Taylor series around $q^{*}$ up to the second-order term.
} 
Thus, the predictor function is convex whenever the following inequality is satisfied:

$$
1>\frac{(1-1 / 2 \chi)}{q(1-1 / 2 \chi)+1 / 2 \chi q_{n+1}^{\mathrm{tr}}}\left(q+\alpha p_{n+1}^{\mathrm{tr}}\right),
$$

which, upon rearranging, gives

$$
\frac{1}{2} \chi\left(q_{n+1}^{\mathrm{tr}}+\alpha p_{n+1}^{\mathrm{tr}}\right)-\alpha p_{n+1}^{\mathrm{tr}}>0 .
$$

It follows from the preceding equation that the convexity condition cannot be guaranteed for any admissible values of the trial stress and the material parameter $\chi$. Only for $p_{n+1}^{\mathrm{tr}}<0$ the above inequality holds regardless of the value of $\chi$.

\section{APPENDIX B: DERIVATION OF THE IMPL-EX ALGORITHMIC ELASTOPLASTIC TANGENT MODULI}

The spatial algorithmic tangent moduli is the fourth-order tensor satisfying:

$$
\mathscr{L}_{v} \tilde{\tau}_{n+1}=\tilde{\boldsymbol{c}}_{\boldsymbol{e p}, n+1}: \mathscr{L}_{v} \mathbf{e}_{n+1}=\tilde{\boldsymbol{c}}_{\boldsymbol{e p}, n+1}: \mathbf{d}_{n+1} .
$$

Taking the Lie derivative of the constitutive relation $\tilde{\boldsymbol{\tau}}_{n+1}=\tilde{\boldsymbol{c}}_{\boldsymbol{e}, n+1}: \tilde{\mathbf{e}}_{e, n+1}$, we obtain

$$
\mathscr{L}_{v} \tilde{\tau}_{n+1}=\mathscr{L}_{v} \tilde{\boldsymbol{c}}_{\boldsymbol{e}, n+1}: \tilde{\mathbf{e}}_{e, n+1}+\tilde{\boldsymbol{c}}_{\boldsymbol{e}, n+1}:\left(\mathbf{d}_{n+1}-\mathscr{L}_{v} \tilde{\mathbf{e}}_{p, n+1}\right) .
$$

The derivation of an expression for the Lie derivative of tensor $\tilde{\boldsymbol{c}}_{\boldsymbol{e}, n+1}$ is accomplished by applying systematically the standard product rule-that also holds for the Lie derivative, i.e. $\mathscr{L}_{v}(A \cdot B)=$ $\mathscr{L}_{v} A \cdot B+A \cdot \mathscr{L}_{v} B-$ on the components of the elasticity tensor (see (4)). Considering that the Lie derivative of the metric tensor ${ }^{+*}$ is, in component form, $\left(\mathscr{L}_{v} \mathbf{g}\right)_{* a b}=2 d_{a b}$, it can be shown that the Lie derivative of the (fully contravariant) fourth-order volumetric tensor $\left.\left(\boldsymbol{I}_{\mathrm{vol}}\right)^{a b c d}=\mathbf{g}^{-1} \otimes \mathbf{g}^{-1}\right)^{a b c d}$ is given by:

$$
\left(\mathscr{L}_{v} \boldsymbol{I}_{\mathrm{vol}}\right)^{a b c d} e_{c d}=-2\left(\operatorname{tr} \mathbf{e}(\boldsymbol{I})^{a b e f}+g^{a b} e^{e f}\right) d_{e f} .
$$

The same lines that lead to the above equation can be followed to deduce the expression for the Lie derivative of the (fully contravariant) fourth order, symmetric identity tensor:

$$
\left(\mathscr{L}_{v} \boldsymbol{I}_{\mathrm{sym}}\right)^{a b c d} e_{c d}=-2\left(e^{a f} g^{b e}+g^{a e} e^{b f}\right) d_{e f},
$$

From identities (B3) and (B4), and after some algebra, one finally arrives at

$$
\tilde{\mathbf{a}}_{\mathbf{e}, n+1}\left(\tilde{\mathbf{e}}_{e, n+1}\right)=-2\left(\left(\tilde{\kappa}_{n+1}^{e}-\frac{2}{3} \tilde{\mu}_{n+1}^{e}\right)\left(\mathbf{1} \otimes \tilde{\mathbf{e}}_{e, n+1}+\operatorname{tr} \tilde{\mathbf{e}}_{e, n+1} \boldsymbol{I}\right)+2 \tilde{\mu}_{n+1}^{e} \mathscr{I}_{s}\left(\tilde{\mathbf{e}}_{e, n+1}\right)\right),
$$

where the operator $\mathscr{I}_{S}(\bullet)$ has the following component form:

$$
\left(\mathscr{I}_{s}(\bullet)\right)^{a b e f}=\frac{1}{2}\left((\bullet)^{a f} \delta^{b e}+(\bullet)^{b f} \delta^{a e}\right) .
$$

\footnotetext{
$\$+$ In a cartesian coordinates system, $g_{a b}=\delta_{a b}$, being $\delta_{a b}$ the second-order Kronecker delta. Index convention used throughout this appendix, on the other hand, follows [35]: upper case letters refer to the reference configuration $\Omega_{0}$ and lower case for the current configuration $\Omega_{t}$; index placement (superindex or subindex) indicates the contravariant or covariant nature, respectively, of the corresponding tensors.
} 
The rightmost term in (B2) can be expanded using Koiter's rule:

$$
\begin{aligned}
\mathscr{L}_{v} \tilde{\mathbf{e}}_{p, n+1} & =\mathscr{L}_{v} \breve{\mathbf{e}}_{p, n}+\overbrace{\sum_{\beta=1}^{3} \frac{\partial \Delta \tilde{\lambda}_{n+1}^{\beta}}{\partial t_{n+1}}}^{=0} \tilde{\boldsymbol{m}}_{n+1}^{\beta}+\sum_{\beta=1}^{3} \Delta \tilde{\lambda}_{n+1}^{\beta} \mathscr{L}_{v} \tilde{\boldsymbol{m}}_{n+1}^{\beta} \\
& =\mathbf{F}_{n+1} \cdot \overbrace{\frac{\partial \mathbf{E}_{p, n}}{\partial t_{n+1}} \cdot \mathbf{F}_{n+1}^{T}+\sum_{\beta=1}^{3} \Delta \tilde{\lambda}_{n+1}^{\beta} \mathscr{L}_{v} \tilde{\boldsymbol{m}}_{n+1}^{\beta}} \\
& =\sum_{\beta=1}^{3} \Delta \tilde{\lambda}_{n+1}^{\beta} \mathscr{L}_{v} \tilde{\boldsymbol{m}}_{n+1}^{\beta} .
\end{aligned}
$$

Hence, the task of obtaining the Lie derivative of $\tilde{\mathbf{e}}_{p, n+1}$ reduces to finding the Lie derivative of the plastic flow vectors associated to each surface. Applying the Lie derivative to the affine transformation (78) relating the plastic flow vector and the Kirchhoff stress tensor yields

$$
\begin{aligned}
\mathscr{L}_{v} \tilde{\boldsymbol{m}}_{n+1}^{\beta} & =\mathscr{L}_{v}\left(\tilde{\boldsymbol{A}}_{n+1}^{\beta}: \tilde{\tau}_{n+1}\right)+\mathscr{L}_{v} \tilde{\boldsymbol{B}}_{n+1}^{\beta} \\
& =\mathscr{L}_{v} \tilde{\boldsymbol{A}}_{n+1}^{\beta}: \tilde{\tau}_{n+1}+\tilde{\boldsymbol{A}}_{n+1}^{\beta}: \mathscr{L}_{v} \tilde{\tau}_{n+1}+\mathscr{L}_{v} \tilde{\boldsymbol{B}}_{n+1}^{\beta} .
\end{aligned}
$$

The procedure for determining the Lie derivative of $\tilde{\boldsymbol{A}}_{n+1}^{\beta}$ parallels that followed in arriving at (B5) $^{\S \S \S}$; the double contraction of $\tilde{\boldsymbol{A}}_{n+1}^{\beta}$ and the contravariant Kirchhoff stress $\tilde{\tau}_{n+1}$ ) is expressible as:

$$
\mathscr{L}_{v} \tilde{\boldsymbol{A}}_{n+1}^{\beta}: \tilde{\tau}_{n+1}=\mathbf{a}_{\tau}\left(\tilde{u}_{n+1}^{\beta}, \tilde{\tau}_{n+1}\right): \mathbf{d}_{n+1},
$$

where

$$
\mathbf{a}_{\tau}\left(\tilde{u}_{n+1}^{\beta}, \tilde{\tau}_{n+1}\right)=2\left(\frac{1}{3}\left(\tilde{u}_{n+1}^{\beta}-2\right)\left(\mathbf{1} \otimes \tilde{\tau}_{n+1}+\operatorname{tr} \tilde{\tau}_{n+1} \boldsymbol{I}\right)+4 \mathscr{I}_{s}\left(\tilde{\tau}_{n+1}\right)\right) .
$$

The remaining term in (B8) is given by

$$
\mathscr{L}_{v} \tilde{\boldsymbol{B}}_{n+1}^{\beta}=\frac{\partial \tilde{v}_{n+1}^{\beta}}{\partial t_{n+1}} \mathbf{1}+\tilde{v}_{n+1}^{\beta} \mathscr{L}_{v} \mathbf{1} .
$$

The first term on the right-hand side of the above equation is zero, since $\tilde{v}_{n+1}^{\beta}$ depends upon $\tilde{\xi}_{n+1}^{h}$ and $\tilde{\xi}_{n+1}^{h}$. The second term involves the Lie derivative of the covariant identity tensor, that is, the metric tensor. Thus, Equation (B11) can be written as

$$
\mathscr{L}_{v} \tilde{\boldsymbol{B}}_{n+1}^{\beta}=2 \tilde{v}_{n+1}^{\beta} \mathbf{d}_{n+1} .
$$

The derivative $\mathscr{L}_{v} \tilde{\mathbf{e}}_{p, n+1}$ is then obtained by combining Equations (B12), (B9) and (B8):

$$
\mathscr{L}_{v} \tilde{\mathbf{e}}_{p, n+1}=\left(\sum_{\beta=1}^{3} \Delta \tilde{\lambda}_{n+1}^{\beta}\left(\mathbf{a}_{\tau}\left(\tilde{u}_{n+1}^{\beta}, \tilde{\tau}_{n+1}\right)+2 \tilde{v}_{n+1}^{\beta}\right)\right): \mathbf{d}_{n+1}+\left(\sum_{\beta=1}^{3} \Delta \tilde{\lambda}_{n+1}^{\beta} \tilde{\boldsymbol{A}}_{n+1}^{\beta}\right): \mathscr{L}_{v} \tilde{\tau}_{n+1} .
$$

$\S \S$ The only difference arises from the fact that $\tilde{\boldsymbol{A}}_{n+1}^{\beta}$ is a fully covariant tensor (as distinct from the elasticity tensor $\tilde{\boldsymbol{c}}_{\boldsymbol{e}, n+1}$, which is fully contravariant), since $\tilde{\boldsymbol{m}}_{n+1}^{\beta}$ has the same tensorial character as the plastic strain tensor, that is, $\tilde{\boldsymbol{m}}_{n+1}^{\beta}$ is also covariant. 
After substituting (B13) and (B5) into (B2), and collecting terms involving $\mathscr{L}_{v} \tilde{\tau}_{n+1}$ and $\mathbf{d}_{n+1}$, the expected expression for the spatial algorithmic tangent tensor is finally achieved

$$
\tilde{\boldsymbol{c}}_{\boldsymbol{e p}, n+1}=\tilde{\boldsymbol{R}}_{n+1}^{-1}:\left(\mathbf{a}_{\mathbf{e}}\left(\tilde{\mathbf{e}}_{e, n+1}\right)+\tilde{\boldsymbol{c}}_{\boldsymbol{e}, n+1}:\left(\boldsymbol{I}-\sum_{\beta=1}^{3} \Delta \tilde{\lambda}_{n+1}^{\beta} \tilde{z}_{n+1}^{\beta}\left(\tilde{\tau}_{n+1}\right)\right)\right),
$$

where

$$
\tilde{\boldsymbol{R}}_{n+1}=\boldsymbol{I}+\tilde{\boldsymbol{c}}_{\boldsymbol{e}, n+1}: \sum_{\beta=1}^{3} \Delta \tilde{\lambda}_{n+1}^{\beta} \tilde{\boldsymbol{A}}_{n+1}^{\beta}
$$

and

$$
\tilde{\boldsymbol{z}}_{n+1}^{\beta}\left(\tilde{\tau}_{n+1}\right)=\mathbf{a}_{\tau}\left(\tilde{u}_{n+1}^{\beta}, \tilde{\tau}_{n+1}\right)+2 \tilde{v}_{n+1}^{\beta} \boldsymbol{I} .
$$

\section{ACKNOWLEDGEMENTS}

Both the Spanish Ministry of Science and Innovation and the Catalan Government Research Department are gratefully acknowledged for financial support under grants BIA2008-00411 and 2009 SGR 1510, respectively.

\section{REFERENCES}

1. Hernández JA, Oliver J, Cante JC, Weyler R. Numerical modeling of crack formation in powder forming processes. International Journal of Solids and Structures 2011; 48:292-316.

2. Lewis R, Gethin D, Yang X, Rowe R. A combined finite-discrete element method for simulating pharmaceutical powder tableting. International Journal for Numerical Methods in Engineering 2005; 62(7):853-869.

3. Coube O, Riedel H. Numerical simulation of metal powder die compaction with special consideration of cracking. Powder Metallurgy 2000; 43:123-131.

4. Coube O. Modelling and numerical simulation of powder die compaction with consideration of cracking. Ph.D. Thesis, University Pierre et Marie Curie, Paris VI, Paris, 1998.

5. Gu C, Kim M, Anand L. Constitutive equations for metal powders: application to powder forming processes. International Journal of Plasticity 2001; 17:147-209.

6. Kraft T, Coube O, Riedel H. Numerical simulation of pressing and sintering in the ceramic and hard metal industry. Recent Developments in Computer Modeling of Powder Metallurgy Processes. Ios Pr Inc., 2001; 181-189. ISBN: 1586030949.

7. Khoei AR, Lewis RW. Adaptive finite element remeshing in a large deformation analysis of metal powder forming. Internation Journal for Numerical Methods in Engineering 1999; 45:801-820.

8. Oliver J, Oller S, Cante JC. A plasticity model for simulation of industrial powder compaction processes. Journal of Solids and Structures 1996; 33:3161-3178.

9. Chtourou H, Gakwaya A, Guillot M. Modeling of the metal powder compaction process using the cap model. Part II: numerical implementation and practical applications. International Journal of Solids and Structures 2002; 39:1077-1096.

10. Perez-Foguet A, Rodriguez-Ferran A, Huerta A. Efficient and accurate approach for powder compaction problems. Computational Mechanics 2003; 30(3):220-234.

11. Kundu P. Fluid Mechanics (2nd edn). Academic Press: London, 2002.

12. Moran B, Ortiz M, Shih CF. Formulation of implicit finite element methods for multiplicative finite deformation plasticity. International Journal for Numerical Methods in Engineering 1990; 29:483-514.

13. Marin E, McDowell D. A semi-implicit integration scheme for rate-dependent and rate-independent plasticity. Computers and Structures 1997; 63(3):579-600.

14. Belytschko T, Liu WK, Moran B. Nonlinear Finite Elements for Continua and Structures. Wiley: New York, 2001.

15. Oliver J, Huespe AE, Blanco S, Linero DL. Stability and robustness issues in numerical modeling of material failure with the strong discontinuity approach. Computer Methods in Applied Mechanics and Engineering 2006; 195:7093-7114.

16. Tu X, Andrade J, Chen Q. Return mapping for nonsmooth and multiscale elastoplasticity. Computer Methods in Applied Mechanics and Engineering 2009; 198(30-32):2286-2296.

17. Oliver J, Huespe AE, Cante JC. An implicit/explicit integration scheme to increase computability of nonlinear material and contact/friction problems. Computer Methods in Applied Mechanics and Engineering 2008; 197:1865-1889.

18. Simo J, Oliver J, Armero F. An analysis of strong discontinuities induced by strain softening in rate-independent inelastic solids. Computational Mechanics 1993; 12:277-296. 
19. Armero F, Pérez-Foguet A. On the formulation of closest-point projection algorithms in elastoplasticity. Part I: the variational structure. International Journal for Numerical Methods in Engineering 2002; 53(2):297-329.

20. Perez-Foguet A, Armero F. On the formulation of closest-point projection algorithms in elastoplasticity. Part II: globally convergent schemes. International Journal for Numerical Methods in Engineering 2002; 53(2):331-374.

21. Blasco J, Codina R, Huerta A. A fractional-step method for the incompressible Navier-Stokes equations related to a predictor-multicorrector algorithm. International Journal for Numerical Methods in Engineering 1998; 28:1391-1419.

22. Oliver J. On the discrete constitutive models induced by strong discontinuity kinematics and continuum constitutive equations. International Journal of Solids and Structures 2000; 37:7207-7229.

23. Oliver J. A consistent characteristic length for smeared cracking models. International Journal for Numerical Methods in Engineering 1989; 28:461-474.

24. Simo JC. Topics on the numerical analysis and simulation of plasticity. In Handbook of Numerical Analysis, Ciarlet PG, Lions JL (eds), vol. III. Elsevier Science Publisher: Amsterdam, 1999.

25. Quarteroni A, Sacco R, Saleri F. Numerical Mathematics. Springer: New York, 2000.

26. Hoffman JD. Numerical Methods for Engineers and Scientists. Marcel Dekker: New York, 2001.

27. Oliver J, Cante JC, Weyler R, González C, Hernández JA. Particle finite element methods in solid mechanics. Computational Plasticity. Springer: Berlin, 2007.

28. Agelet de Saracibar C, Chiumenti M, Valverde Q, Cervera M. On the orthogonal subgrid scale pressure stabilization of finite deformation j2 plasticity. Computer Methods in Applied Mechanics and Engineering 2006; 195:1224-1251.

29. Cervera M, Chiumenti M, Agelet de Saracibar C. Softening, localization and stabilization: capture of discontinuous solutions in j2 plasticity. International Journal for Numerical and Analytical Methods in Geomechanics 2004; 28:373-393.

30. Hernández J. Numerical modeling of crack formation in powder compaction based manufacturing problems. Ph.D. Thesis, Technical University of Catalonia, Barcelona, 2009.

31. Doremus P, Toussaint F, Alvain O. Simple tests and standard procedure for the characterization of green compacted powder. In Recent Developments in Computer Modeling of Powder Metallurgy Processes, Zavaliangos A, Lapter A (eds). IOS Press: Kiev, Ukraine, 15-18 May 2000; 277-285.

32. Jonsén P, Häggblad HA, Sommer K. Tensile strength and fracture energy of pressed metal powder by diametral compression test. Powder Technology 2007; 176:148-155.

33. Crisfield MA. Non-linear Finite Element Analysis of Solids and Structures, Volume 1: Essentials. Wiley: Chichester, 1991.

34. Nocedal J, Wright SJ. Numerical Optimization. Springer: New York, 1999.

35. Marsden JE, Hugues TJR. Mathematical Foundations of Elasticity. Dover: New York, 1994. 